ESAIM: M2AN 48 (2014) 765-793

DOI: $10.1051 / \mathrm{m} 2 \mathrm{an} / 2013120$
ESAIM: Mathematical Modelling and Numerical Analysis

www.esaim-m2an.org

\title{
NUMERICAL ANALYSIS OF MODULAR REGULARIZATION METHODS FOR THE BDF2 TIME DISCRETIZATION OF THE NAVIER-STOKES EQUATIONS *
}

\author{
William Layton ${ }^{1}$, Nathaniel Mays ${ }^{2}$, Monika Neda $^{3}$ and Catalin Trenchea ${ }^{4}$
}

\begin{abstract}
We consider an uncoupled, modular regularization algorithm for approximation of the Navier-Stokes equations. The method is: Step 1.1: Advance the NSE one time step, Step 1.1: Regularize to obtain the approximation at the new time level. Previous analysis of this approach has been for specific time stepping methods in Step 1.1 and simple stabilizations in Step 1.1. In this report we extend the mathematical support for uncoupled, modular stabilization to (i) the more complex and better performing BDF2 time discretization in Step 1.1, and (ii) more general (linear or nonlinear) regularization operators in Step 1.1. We give a complete stability analysis, derive conditions on the Step 1.1 regularization operator for which the combination has good stabilization effects, characterize the numerical dissipation induced by Step 1.1, prove an asymptotic error estimate incorporating the numerical error of the method used in Step 1.1 and the regularizations consistency error in Step 1.1 and provide numerical tests.
\end{abstract}

Mathematics Subject Classification. 35Q30, 76F65.

Received October 20, 2011. Revised July 3, 2013.

Published online April 1, 2014.

\section{INTRODUCTION}

This report continues the numerical analysis of modular, uncoupled stabilization/regularization methods for (primarily under-resolved) flow problems, extending their analytical foundation from one step Crank-Nicolson $(\mathrm{CN})$ method and linear filtering done in [17] to the multi-step BDF2 time discretization and both linear and nonlinear regularization operators. To begin, for $\Omega$ a polyhedral domain in $\mathbb{R}^{d}, d=2,3$, the fluid velocity $u(x, t)$

Keywords and phrases. Modular regularization, BDF2 time discretization, Navier-Stokes equations, turbulence, finite element method.

* This research was partially supported by the NSF under grants DMS-0810385 and DMS-126465, and by the Air Force under grant FA9550-12-1-0191.

1 Department of Mathematics, University of Pittsburgh, Pittsburgh, PA, 15260, USA. wjl@pitt.edu

2 Department of Mathematics, Wheeling Jesuit University, Wheeling, WV, 26003, USA. nmays@wju.edu

3 Department of Mathematical Sciences, University of Nevada Las Vegas, USA. Monika.Neda@unlv.edu

4 Department of Mathematics, University of Pittsburgh, Pittsburgh, PA, 15260, USA. trenchea@pitt.edu 
and pressure $p(x, t)$ satisfy:

$$
\begin{aligned}
& u_{t}+u \cdot \nabla u-\nu \triangle u+\nabla p=f(x, t) \text { and } \nabla \cdot u=0, \text { in } \Omega \times(0, T], \\
& u=0 \quad \text { on } \partial \Omega \text { and } u(x, 0)=u^{0}(x) \text { in } \Omega .
\end{aligned}
$$

The frequent necessity of underresolved flow simulations has led to numerous regularizations and stabilizations in Computational Fluid Dynamics. Regularizations based on "evolve one time step then regularize" fits well with the modular development of complex codes and with legacy codes, and has been studied by Boyd [7], Fischer and Mullen [18,33], Mathew et al. [32], Dunca [12], as well as [16,30,31]. Numerical analysis of the stability, dissipation and error behaviour in linear filter based stabilization of the Crank-Nicolson method with finite element discretization was performed in [17], including effects of deconvolution and relaxation. The case of backward Euler time discretization plus nonlinear filtering, and relaxation was considered in [30]. Mathew, Lechner, Foysi, Sesterhenn and Friedrich [32] pointed out that this stabilization induces a new implicit time relaxation term that acts to damp oscillations in marginally resolved scales. See also Section 5.3.3 in Garnier, Adams and Sagaut [19] and Visbal and Rizzetta [46]. The connection to time relaxation links the methods herein to work of Schochet and Tadmor [37], Roseneau [35], Adams, Kleiser, Leonard and Stolz [1-3, 39-42], Dunca [12-14] and Layton, Neda, Manica, Rebholz, Ervin and Connors [11,16, 26, 28, 29].

To present the method, let $X=\left(H_{0}^{1}(\Omega)\right)^{d}, Q=L_{0}^{2}(\Omega)$; we let $X_{h} \subset X, Q_{h} \subset Q$ denote velocity, pressure finite element spaces satisfying the usual discrete inf-sup condition, see Section 2 for full details. Let $V_{h} \subset X_{h}$ denote the discretely divergence free subspace of $X_{h}$. We shall denote the regularization operator by the, possibly nonlinear, map $G_{h}: X \rightarrow V_{h}$. We give five examples of computationally attractive $G_{h}(\cdot)$ satisfying (1.3) in Section 2.2 .

Algorithm 1.1 (BDF2, Regularize, Relax for NSE).

Choose $\chi$ with $0 \leq \chi \leq 1$.

Step 1: Given $u_{h}^{n}, u_{h}^{n-1}$ find $w_{h}^{n+1} \in X_{h}, p_{h}^{n+1} \in Q_{h}$ satisfying

$$
\begin{aligned}
& \left(\frac{3 w_{h}^{n+1}-4 u_{h}^{n}+u_{h}^{n-1}}{2 \triangle t}, v_{h}\right)+b^{*}\left(w_{h}^{n+1}, w_{h}^{n+1}, v_{h}\right)+\nu\left(\nabla w_{h}^{n+1}, \nabla v_{h}\right)-\left(p_{h}^{n+1}, \nabla \cdot v_{h}\right)=\left(f^{n+1}, v_{h}\right), \forall v_{h} \in X_{h}, \\
& \left(\nabla \cdot w_{h}^{n+1}, q_{h}\right)=0, \forall q_{h} \in Q_{h} .
\end{aligned}
$$

Step 2: (a) Regularize $w_{h}^{n+1}$ to give $G_{h}\left(w_{h}^{n+1}\right)$, and

(b) relax

$$
u_{h}^{n+1}=(1-\chi) w_{h}^{n+1}+\chi G_{h}\left(w_{h}^{n+1}\right) .
$$

Step 1.1, without Step 1.1 of Algorithm 1.1 is the classical BDF2-FEM (finite element method) discretization of the Navier-Stokes equations analyzed in [4] (under a small data condition) and [15,34,47]. The relaxation in (1.2) in Step 1.1 with the typical choice $\chi=\Delta t$, was introduced by Fischer and Mullen in [18,33] to keep numerical diffusion from blowing up as $\Delta t \rightarrow 0$.

This report gives a comprehensive stability and convergence analysis of Algorithm 1.1 for general $G_{h}(\cdot)$, including low and high order filters, nonlinear filters, deconvolution based regularizations and others. This analysis shows, Section 3, that the key requirements for unconditional stability are: $0 \leq \chi \leq 1$ and

$$
\left(G_{h}(v), v\right)>0 \quad \text { and } \quad\left(v-G_{h}(v), G_{h}(v)\right)>0 \quad \text { for all } v \neq 0 .
$$

If we denote the regularization's consistency error by

$$
\epsilon(u):=\left\|u-G_{h}(u)\right\|
$$

then the temporal consistency error of Algorithm 1.1 is $O\left(\Delta t^{3}+\chi \epsilon(u)\right)$ which forecasts a global error of $O\left(\Delta t^{2}+\frac{\chi}{\Delta t} \epsilon(u)+\right.$ spatial FEM error). We prove that under (1.3) this error is indeed attained. Section 5 presents tests of Algorithm 1.1 of underresolved flows. 


\section{Preliminaries}

The $L^{2}(\Omega)$ norm and inner product will be denoted by $\|\cdot\|$ and $(\cdot, \cdot)$. The $L^{p}(\Omega)$ and $W_{p}^{k}(\Omega)$ norms are denoted by $\|\cdot\|_{L^{p}}$ and $\|\cdot\|_{W_{p}^{k}}$, respectively and the semi-norm by $|\cdot|_{W_{p}^{k}} \cdot H^{k}$ denotes $W_{2}^{k}(\Omega)$, and $\|\cdot\|_{k}$ the norm in $H^{k}$. The space $H^{-k}$ denotes the dual space of $H_{0}^{k}$. For functions $v(x, t)$ define

$$
\|v\|_{\infty, k}:=\operatorname{EssSup}_{[0, T]}\|v(t, \cdot)\|_{k}, \text { and }\|v\|_{m, k}:=\left(\int_{0}^{T}\|v(t, \cdot)\|_{k}^{m} \mathrm{~d} t\right)^{1 / m} .
$$

Let $X:=\left(H_{0}^{1}(\Omega)\right)^{d}, Q:=L_{0}^{2}(\Omega)$. We shall assume that the solution to the NSE is a strong solution and satisfies

$$
\begin{aligned}
& u \in L^{2}(0, T ; X) \cap L^{\infty}\left(0, T ; L^{2}(\Omega)\right) \cap L^{4}(0, T ; X), \\
& p \in L^{2}(0, T ; Q), \quad u_{t} \in L^{2}\left(0, T ; X^{*}\right),
\end{aligned}
$$

and

$$
\begin{aligned}
\left(u_{t}, v\right)+(u \cdot \nabla u, v)-(p, \nabla \cdot v)+\nu(\nabla u, \nabla v) & =(f, v) \quad \forall v \in X, \\
(\nabla \cdot u, q) & =0 \quad \forall q \in Q .
\end{aligned}
$$

We renorm $X$ by $\|v\|_{X}:=\|\nabla v\|$ which, because of the boundary condition, is a norm. The space of divergence free functions is given by

$$
V:=\{v \in X:(\nabla \cdot v, q)=0 \quad \forall q \in Q\} .
$$

We shall denote conforming velocity-pressure finite element spaces based on an edge to edge triangulation of $\Omega$ (with maximum triangle diameter $h$ ) by

$$
X_{h} \subset X, Q_{h} \subset Q .
$$

We shall assume that $\left(X_{h}, Q_{h}\right)$ satisfy the usual inf-sup condition necessary for the stability of the pressure, e.g. $[20,21]$ and that the usual approximation properties of piecewise polynomials of degree $k, k-1$ hold for $\left(X_{h}, Q_{h}\right)$. The discretely divergence free subspace of $X_{h}$ is

$$
V_{h}=\left\{v_{h} \in X_{h}:\left(\nabla \cdot v_{h}, q_{h}\right)=0 \quad \forall q_{h} \in Q_{h}\right\} .
$$

Taylor-Hood elements (see e.g. $[8,20]$ ) are one common example of such a choice with $k=2$ for $\left(X_{h}, Q_{h}\right)$, and are also the elements we use in our numerical experiments. Define the usual explicitly skew symmetrized trilinear form

$$
b^{*}(u, v, w):=\frac{1}{2}(u \cdot \nabla v, w)-\frac{1}{2}(u \cdot \nabla w, v) .
$$

To set notation, let

$$
t^{n}=n \Delta t, n=0,1,2, \ldots, N_{T}, T:=N_{T} \Delta t, \quad \text { and } \quad d_{t} f^{n}:=\frac{f\left(t^{n}\right)-f\left(t^{n-1}\right)}{\Delta t} .
$$

Introduce the following discrete norms

$$
\||v|\|_{\infty, k}:=\max _{0 \leq n \leq N_{T}}\left\|v^{n}\right\|_{k}, \quad\|v\|_{m, k}:=\left(\Delta t \sum_{n=0}^{N_{T}}\left\|v^{n}\right\|_{k}^{m}\right)^{1 / m} .
$$




\subsection{Regularization operators}

Proposition 2.1. Under assumptions (1.3), the regularization map $G_{h}$ satisfies additionally: for all $v \in X$

$$
\begin{aligned}
& 0<\left(v-G_{h}(v), v\right), \quad v \neq 0, \\
& \left(G_{h}(v), v\right) \leq\|v\|^{2}, \\
& \left(v-G_{h}(v), v\right) \leq\|v\|^{2} .
\end{aligned}
$$

Proof. For all $v \in X$ we have that

$$
\left(v-G_{h}(v), v\right)=\left\|v-G_{h}(v)\right\|^{2}+\left(v-G_{h}(v), G_{h}(v)\right) \geq\left(v-G_{h}(v), G_{h}(v)\right)
$$

and (2.5) follows immediately from (1.3). To prove the second claim, note that

$$
\left(G_{h}(v), v\right)=\|v\|^{2}-\left(v-G_{h}(v), v\right)
$$

and use (2.5). Finally, by (1.3) we obtain

$$
\left(v-G_{h}(v), v\right)=\|v\|^{2}-\left(G_{h}(v), v\right) \leq\|v\|^{2} .
$$

The work of computing the action of a general, nonlinear regularization operator $G(\phi)$ can be considerable. We shall thus restrict $G_{h}(\cdot)$ to be semilinear (Assumption 2.2) satisfying a uniform positivity assumption (Assumption 2.3) below.

\section{Assumptions 2.2.}

$$
G_{h}(\phi)=\mathcal{G}_{h}(\phi) \phi
$$

where $\forall w \in X, \mathcal{G}_{h}(w) \in \mathcal{L}\left(V_{h}, V_{h}\right)$ is a linear and continuous operator.

Assumptions 2.3. For any $w \in X$, the linear operator $\mathcal{G}_{h}(w) \in \mathcal{L}\left(V_{h}, V_{h}\right)$ satisfies

$$
\begin{aligned}
& \left(\mathcal{G}_{h}(w) \phi, \phi\right)>0 \text { for all } \phi \neq 0, \\
& \left(\phi-\mathcal{G}_{h}(w) \phi, \mathcal{G}_{h}(w) \phi\right)>0 \text { for all } \phi \neq 0 .
\end{aligned}
$$

Assumption 2.2 means, given $\widehat{\phi}$, computing the action $\phi \rightarrow \mathcal{G}_{h}(\widehat{\phi}) \phi$ (even for $\widehat{\phi}=\phi$ ) requires linear work. This restriction includes the case of $\mathcal{G}_{h}(\cdot)$ being fixed linear operator (e.g. a linear filter) and also plays a key role in the error analysis for nonlinear filters.

Uniform positivity, Assumption 2.3, implies positivity, (1.3), and for linear regularizations they are equivalent. Following the proof of Proposition 2.1, we note that, under Assumption 2.3, $\mathcal{G}_{h}(w)$ is non-expansive and

$$
0<\left(\left(I-\mathcal{G}_{h}(w)\right) v, v\right) \leq\|v\|^{2} \text { for all } v \neq 0 .
$$

\subsection{Examples of regularization operators}

Here $\delta>0$ denotes a regularization length scale and $\phi \in L^{2}(\Omega)$.

\subsubsection{Discrete differential filter.}

The discrete differential filter $G_{h}: L^{2}(\Omega) \rightarrow X_{h}$, is given as $G_{h}(\phi):=\bar{\phi}_{h}$, where $\bar{\phi}_{h} \in X_{h}$ is the unique solution of

$$
\delta^{2}\left(\nabla \bar{\phi}_{h}, \nabla v_{h}\right)+\left(\bar{\phi}_{h}, v_{h}\right)=\left(\phi, v_{h}\right) \quad \forall v_{h} \in X_{h}
$$




\subsubsection{Discrete Stokes differential filter.}

The discrete Stokes differential filter preserves discrete incompressibility. $G_{h}: X^{*} \rightarrow X_{h}$, and $\bar{\phi}_{h}=G_{h}(\phi)$ where $\left(\bar{\phi}_{h}, \rho\right) \in X_{h} \times Q_{h}$ is the unique solution of

$$
\begin{aligned}
\delta^{2}\left(\nabla \bar{\phi}_{h}, \nabla v_{h}\right)+\left(\bar{\phi}_{h}, v_{h}\right)-\left(\rho, \nabla \cdot v_{h}\right) & =\left(\phi, v_{h}\right) \quad \forall v_{h} \in X_{h}, \\
\left(\nabla \cdot \bar{\phi}_{h}, q\right) & =0 \quad \forall q \in Q_{h} .
\end{aligned}
$$

The choices (2.11) and (2.12) of $G_{h}$ satisfy (1.3), with regularization error (e.g. Lem. 2.1 in [17])

$$
\epsilon(\phi)+\delta^{2}\left\|\nabla\left(\phi-G_{h}(\phi)\right)\right\|^{2} \leq C \inf _{v_{h} \in V_{h}}\left\{\delta^{2}\left\|\nabla\left(\phi-v_{h}\right)\right\|^{2}+\left\|\phi-v_{h}\right\|^{2}\right\}+C \delta^{4}\|\Delta \phi\|^{2} .
$$

\subsubsection{Nonlinear filters.}

Select a smooth function $a: X \rightarrow \mathbb{R}, a=a(\phi, \nabla \phi, \cdots)$ (denoted by $a(\phi))$ with the properties

$$
0<a_{\min } \leq a(\phi) \leq 1 \quad \text { for any } \phi \in V,
$$

see [30] for examples of such $a(\cdot)$. Define $G_{h}(\phi):=\bar{\phi}_{h}$ as the unique solution of: find $\left(\bar{\phi}_{h}, \lambda_{h}\right) \in X_{h} \times Q_{h}$ satisfying

$$
\begin{aligned}
\left(\delta^{2} a(\phi) \nabla \bar{\phi}^{h}, \nabla v_{h}\right)+\left(\bar{\phi}^{h}, v_{h}\right)-\left(\lambda_{h}, \nabla \cdot v_{h}\right) & =\left(\phi, v_{h}\right) \quad \forall v_{h} \in X_{h}, \\
\left(\nabla \cdot \bar{\phi}^{h}, q\right) & =0 \quad \forall q \in Q_{h} .
\end{aligned}
$$

Note that $\bar{\phi}_{h}:=G_{h}(\phi)=\mathcal{G}_{h}(\phi) \phi$, where the linear and continuous operator $\mathcal{G}_{h}(w) \phi:=\widetilde{\phi}$ is the unique solution $\left(\widetilde{\phi}_{h}, \lambda_{h}\right) \in X_{h} \times Q_{h}$ of

$$
\begin{aligned}
\left(\delta^{2} a(w) \nabla \widetilde{\phi}^{h}, \nabla v_{h}\right)+\left(\widetilde{\phi}^{h}, v_{h}\right)-\left(\lambda_{h}, \nabla \cdot v_{h}\right) & =\left(\phi, v_{h}\right) \quad \forall v_{h} \in X_{h}, \\
\left(\nabla \cdot \widetilde{\phi}^{h}, q\right) & =0 \quad \forall q \in Q_{h} .
\end{aligned}
$$

Lemma 2.4. Let $G_{h}(\cdot)$ be the nonlinear filter (2.13)-(2.14). Assumption 2.3 holds. Thus (1.3) holds as well: for all $\phi \in V_{h}$ we have

$$
\begin{array}{lll}
\left(G_{h}(\phi), \phi\right)>0, & \text { and } & \left(\mathcal{G}_{h}(w) \phi, \phi\right)>0 \quad \text { for all } w, \phi \neq 0 \\
\left(\phi-G_{h}(\phi), G_{h}(\phi)\right)>0, & & \left(\phi-\mathcal{G}_{h}(w) \phi, \mathcal{G}_{h}(w) \phi\right)>0 \quad \text { for all } w, \phi \neq 0 .
\end{array}
$$

Proof. It is sufficient to prove that any $w \in X, \mathcal{G}_{h}(w)$ satisfies (2.8), (2.9). For $v_{h} \in V_{h}(2.15)-(2.16)$ are equivalent to:

$$
\left(\delta^{2} a(w) \nabla \widetilde{\phi}_{h}, \nabla v_{h}\right)+\left(\widetilde{\phi}_{h}, v_{h}\right)=\left(\phi, v_{h}\right) \quad \forall v_{h} \in V_{h} .
$$

To prove the first assertion, set $v_{h}=\widetilde{\phi}_{h}$. We then have

$$
\left(\mathcal{G}_{h}(w) \phi, \phi\right)=\left(\phi, \widetilde{\phi}_{h}\right)=\int_{\Omega} \delta^{2} a(w)\left|\nabla \widetilde{\phi}_{h}\right|^{2}+\left|\widetilde{\phi}_{h}\right|^{2} .
$$

For the second claim, note that

$$
\left(\phi-\widetilde{\phi}_{h}, \widetilde{\phi}_{h}\right)=\int_{\Omega} \delta^{2} a(w)\left|\nabla \widetilde{\phi}^{h}\right|^{2} \mathrm{~d} x \geq \delta^{2} a_{\min }\left\|\nabla \widetilde{\phi}_{h}\right\|^{2} .
$$

This is positive for $\phi \neq 0$. Indeed, if $\left\|\nabla \widetilde{\phi}_{h}\right\|^{2}=0$, then $\widetilde{\phi}^{h} \equiv 0$ (due to the zero boundary conditions) and then $\left(\phi, v_{h}\right)=0 \quad \forall v_{h} \in V_{h}$. 
It has been shown in [30] that the nonlinear filter (2.13)-(2.14) has the following regularization error.

Theorem 2.5. Let $X_{h}, Q_{h}$ satisfy the inf-sup condition and $\phi \in V$. Then the discrete nonlinear filter $G_{h}(\phi)$ given by (2.13)-(2.14) satisfies

$\epsilon(\phi)+\int_{\Omega} \delta^{2} a(\phi)\left|\nabla\left(\phi-G_{h}(\phi)\right)\right|^{2} \mathrm{~d} x \leq C \inf _{\hat{\phi} \in V_{h}}\left\{\int_{\Omega} \delta^{2} a(\phi)|\nabla(\phi-\widehat{\phi})|^{2} \mathrm{~d} x+\|\phi-\widehat{\phi}\|^{2}\right\}+C \delta_{\max }^{4}\|\nabla \cdot(a(\phi) \nabla \phi)\|^{2}$.

For the frozen nonlinearity discrete nonlinear filter (2.15)-(2.16): for any given $w_{h} \in V_{h}$, we have that $\forall \phi \in V$

$$
\begin{aligned}
& \int_{\Omega} \delta^{2} a\left(w_{h}\right)\left|\nabla\left(\phi-\mathcal{G}_{h}\left(w_{h}\right) \phi\right)\right|^{2} \mathrm{~d} x+\left\|\phi-\mathcal{G}_{h}\left(w_{h}\right) \phi\right\|^{2} \\
& \leq C \inf _{\widehat{\phi} \in V_{h}}\left\{\int_{\Omega} \delta^{2} a\left(w_{h}\right)|\nabla(\phi-\widehat{\phi})|^{2} \mathrm{~d} x+\|\phi-\widehat{\phi}\|^{2}\right\}+C \delta^{2} \min \left\{\|\nabla \phi\|^{2}, \delta^{2}\left\|\nabla \cdot\left(a\left(w_{h}\right) \nabla \phi\right)\right\|^{2}\right\} .
\end{aligned}
$$

\subsubsection{Modular VMS methods.}

Let $X_{H} \subset X_{h}, Q_{H} \subset Q_{h}$ denote subspaces of the velocity-pressure FEM spaces associated typically with either lower degree polynomials on the same mesh or the same finite element spaces on a coarser mesh. Define $P_{H}: \nabla X_{h} \rightarrow \nabla X_{H}$ to be the $L^{2}$ projection operator. Let $\nu_{T}$ be a bounded, positive, elementwise constant, eddy viscosity parametrization and $\delta>0$ the filter lengthscale. The modular VMS regularization operator introduced in [31] is the linear operator $G_{h}(\phi)=\bar{\phi} \in V_{h}$, the solution of

$$
\left(\nu_{T}\left[I-P_{H}\right] \nabla \bar{\phi},\left[I-P_{H}\right] \nabla v_{h}\right)+\left(\bar{\phi}, v_{h}\right)=\left(\phi, v_{h}\right) \text { for all } v_{h} \in V_{h} .
$$

Lemma 2.6. $G_{h}(\cdot)$, defined by (2.18), satisfies (1.3): for all $\phi \in X_{h}$

$$
\left(G_{h}(\phi), \phi\right)>0 \quad \text { and } \quad\left(\phi-G_{h}(\phi), G_{h}(\phi)\right) \geq 0 .
$$

Proof. The proof is similar to the Lemma 2.4. For the first claim set $v_{h}=\bar{\phi}$ in (2.18). For the second claim set $v_{h}=\bar{\phi}$ again. We have

$$
(\phi-\bar{\phi}, \bar{\phi})=\int_{\Omega} \nu_{T}\left|\left[I-P_{H}\right] \nabla \bar{\phi}\right|^{2} \mathrm{~d} x \geq 0
$$

Note that $\nabla \bar{\phi}$ is the $L^{2}$ projection into $\nabla X_{H}$, thus provided $\phi \in H^{2}(\Omega)$

$$
\epsilon(\phi)=\left\|\phi-G_{h}(\phi)\right\| \leq C H\|\nabla \phi\| .
$$

\subsubsection{Approximate Deconvolution.}

One rich source of high accuracy regularization operators and higher order filters is approximate deconvolution of a filter $F_{h}$, (such as the filters in examples 1 and 2). The van Cittert deconvolution operator is defined by repeated application of a simpler filter $F_{h}$ as follows.

Definition 2.7 (Discrete van Cittert deconvolution). Let $\bar{\phi}=F_{h}(\phi)$ be a linear filter satisfying Assumption 2.3. Then the $N$ th discrete van Cittert operator is:

$$
D_{h}^{N} \phi:=\sum_{n=0}^{N}\left(I-F_{h}\right)^{n} \phi .
$$

We then define $G_{h}(\phi)=D_{h}^{N} \bar{\phi}$. In [17] the conditions (1.3) were proven for $F_{h}$ being the discrete Stokes filter. 
Lemma 2.8. We have $D_{h}^{N}: V_{h} \rightarrow V_{h}$ and for all $\phi \in V_{h}$

$$
\begin{gathered}
\left(D_{h}^{N}(\bar{\phi}), \phi\right)>0 \text { and }\left(\phi-D_{h}^{N}(\bar{\phi}), D_{h}^{N}(\bar{\phi})\right)>0 \text { if } \phi \neq 0, \\
\left\|D_{h}^{N}(\bar{\phi})\right\| \leq\|\phi\| \text { and }\left\|\phi-D_{h}^{N}(\bar{\phi})\right\| \leq\|\phi\| .
\end{gathered}
$$

Proof. Using the symmetry and linearity of $F_{h}$, and Assumption 2.3 for $F_{h}$, we have

$$
\begin{aligned}
& \left(D_{h}^{N}(\bar{\phi}), \phi\right)=\sum_{i=0}^{\left[\frac{N}{2}-1\right]}\left(\left[\left(I-F_{h}\right)^{2 i}+\left(I-F_{h}\right)^{2 i+1}\right](\bar{\phi}), \phi\right)+\left(1-2\left\{\frac{N}{2}\right\}\right)\left(\left(I-F_{h}\right)^{N}(\bar{\phi}), \phi\right) \\
& =\sum_{i=0}^{\left[\frac{N}{2}-1\right]}\left(2\left(\left(I-F_{h}\right)\left(I-F_{h}\right)^{i} \phi, F_{h}\left(I-F_{h}\right)^{i} \phi\right)+\left\|F_{h}\left(I-F_{h}\right)^{i} \phi\right\|^{2}\right) \\
& \quad+\left(1-2\left\{\frac{N}{2}\right\}\right)\left(F_{h}\left(I-F_{h}\right)^{\left[\frac{N}{2}\right]} \phi,\left(I-F_{h}\right)^{\left[\frac{N}{2}\right]} \phi\right)>0 .
\end{aligned}
$$

To prove the second estimate, we note first that $\phi-D_{h}^{N}(\bar{\phi})=\left(I-F_{h}\right)^{N+1} \phi$, and therefore

$$
\left(\phi-D_{h}^{N}(\bar{\phi}), D_{h}^{N}(\bar{\phi})\right)=\sum_{i=0}^{\left[\frac{N}{2}-1\right]}\left(\left[\left(I-F_{h}\right)^{2 i+N+1}+\left(I-F_{h}\right)^{2 i+N+2}\right](\bar{\phi}), \phi\right)+\left(1-2\left\{\frac{N}{2}\right\}\right)\left(\left(I-F_{h}\right)^{2 N+1}(\bar{\phi}), \phi\right)>0 .
$$

The last two estimates follow as in the proof of Proposition 2.1. (See also [17] and Stanculescu [38].)

We also use the following from [28].

Lemma 2.9. For smooth $\phi$ the discrete Nth order discrete approximate deconvolution regularization operator satisfies for $0 \leq s \leq N$

$$
\epsilon(\phi)=\left\|\phi-D_{h}^{N}(\bar{\phi})\right\| \leq C_{1} \delta^{2 s+2}\|\bar{\phi}\|_{H^{2 s+2}}+C_{2}\left(\delta h^{k}+h^{k+1}\right) \sum_{n=1}^{N+1}\left|F_{h}^{n}(\phi)\right|_{k+1} .
$$

Suppose $\phi \in H_{0}^{1}(\Omega) \cap H^{4}(\Omega)$. If $N=1$ and $\left(X_{h}, Q_{h}\right)$ are the Taylor-Hood elements, then

$$
\epsilon(\phi)=\left\|\phi-D_{h}^{N}(\bar{\phi})\right\| \leq C_{1} \delta^{3}\|\phi\|_{3}+C_{2}\left(\delta h^{2}+h^{3}\right)\|\phi\|_{3} .
$$

Proof. For Taylor-Hood elements $(k=2),(2.20)$ follows by (2.19) taking $s=1 / 2, k=2, N=1$ and thus $\left[\frac{k+1}{2}\right]-1=0$. We have then $\|\bar{\phi}\|_{3} \leq C\|\phi\|_{3}$ with uniform constant.

The dependence of the $\left|F_{h}^{n}(\phi)\right|_{k+1}$ terms in (2.19) upon the filter radius $\delta$, for a general smooth function $\phi$, is not fully understood. In the case of $\phi$ periodic the $\left|F_{h}^{n}(\phi)\right|_{k+1}$ are independent of $\delta$. Also, for $\phi$ satisfying homogeneous Dirichlet boundary conditions, with the additional property that $\Delta^{j} \phi=0$ on $\partial \Omega$ for $0 \leq j \leq$ $\left[\frac{k+1}{2}\right]-1$, the $\left|F_{h}^{n}(\phi)\right|_{k+1}$ are also independent of $\delta$, see $[27,28]$.

Motivated by the (2.20), for $N \geq 2$ we assume the following in the convergence analysis.

Assumption DG1: Let $D_{h}^{N}(\cdot)$ be the van Cittert regularization operator. For some $\alpha, 0<\alpha \leq N$

$$
\epsilon(\phi)=\left\|\phi-D_{h}^{N}(\bar{\phi})\right\| \leq C_{1} \delta^{2 \alpha+2}\|\phi\|_{H^{2 \alpha+2}}+C_{2}\left(\delta h^{k}+h^{k+1}\right)\|\phi\|_{k+1} .
$$

\section{Stability of Algorithm 1.1}

We prove an energy equality, unconditional stability and give the numerical dissipation induced in Step 1.1 of Algorithm 1.1. We begin with an algebraic identity. 
Lemma $3.1[17,30]$.

Assume $\chi \in[0,1]$ and let $u_{h}=(1-\chi) w_{h}+\chi G_{h}\left(w_{h}\right)$. Then

$$
\begin{aligned}
& \left\|w_{h}\right\|^{2}-\left\|u_{h}\right\|^{2}=\chi(2-\chi)\left(w_{h}-G_{h}\left(w_{h}\right), w_{h}\right)+\chi^{2}\left(w_{h}-G_{h}\left(w_{h}\right), G_{h}\left(w_{h}\right)\right), \\
& \left\|w_{h}\right\|^{2}-\left\|u_{h}\right\|^{2}=-\left\|u_{h}-w_{h}\right\|^{2}+2 \chi\left(w_{h}-G_{h}\left(w_{h}\right), w_{h}\right) .
\end{aligned}
$$

Proof. The proof follows closely the proof in the case where $G_{h}$ is a linear operator in $[17,30]$.

Definition 3.2. Let $\rho_{\chi, h}: X_{h} \rightarrow \mathbb{R}^{+} \cup\{0\}$ denote the following non-negative functional

$$
\rho_{\chi, h}(v):=\left((1-\chi) v+\chi G_{h}(v), \chi\left(v-G_{h}(v)\right)^{\frac{1}{2}}, \quad \text { for all } v \in X_{h} .\right.
$$

Proposition 2.1 guarantees that, under assumption (1.3), $\rho_{\chi, h}$ is well-defined. While not a norm when $G_{h}(\cdot)$ is nonlinear, when $G_{h}$ is linear, $\rho_{\chi, h}(\cdot)$ is a weighted norm on $X_{h}$. Let also denote

$$
D_{+} D_{-} v^{n-1}=\frac{v^{n}-2 v^{n-1}+v^{n-2}}{\Delta t^{2}}
$$

Proposition 3.3 (Stability).

Under Assumption 2.3, Algorithm 1.1 satisfies the energy equality

$$
\begin{aligned}
& \frac{1}{4}\left\|u_{h}^{n+1}\right\|^{2}+\frac{1}{4}\left\|2 u_{h}^{n+1}-u_{h}^{n}\right\|^{2}+\Delta t \sum_{j=1}^{n} \frac{\Delta t^{3}}{4}\left\|D_{+} D_{-} u_{h}^{j}\right\|^{2}+\Delta t \sum_{j=1}^{n} \frac{3 \chi}{2 \Delta t}\left(w_{h}^{n+1}-G_{h}\left(w_{h}^{n+1}\right), w_{h}^{n+1}\right) \\
& +\frac{1}{4} \rho_{\chi, h}^{2}\left(w_{h}^{n+1}\right)+\frac{1}{4} \rho_{\chi, h}^{2}\left(2 w_{h}^{n+1}-w_{h}^{n}\right)+\Delta t \sum_{j=1}^{n} \frac{\Delta t^{3}}{4} \rho_{\chi, h}^{2}\left(D_{+} D_{-} w_{h}^{j}\right)+\nu \Delta t \sum_{j=1}^{n}\left\|\nabla w_{h}^{n+1}\right\|^{2} \\
& =\frac{1}{4}\left\|u_{h}^{1}\right\|^{2}+\frac{1}{4}\left\|2 u_{h}^{1}-u_{h}^{0}\right\|^{2}+\Delta t \sum_{j=1}^{n}\left(f^{j+1}, w_{h}^{j+1}\right),
\end{aligned}
$$

where the terms $\left(w-G_{h}(w), w\right) \geq 0$, and the stability bound

$$
\begin{aligned}
& \frac{1}{4}\left\|u_{h}^{n+1}\right\|^{2}+\frac{1}{4}\left\|2 u_{h}^{n+1}-u_{h}^{n}\right\|^{2}+\Delta t \sum_{j=1}^{n} \frac{\Delta t^{3}}{4}\left\|D_{+} D_{-} u_{h}^{j}\right\|^{2}+\Delta t \sum_{j=1}^{n} \frac{3 \chi}{2 \Delta t}\left(w_{h}^{j+1}-G_{h}\left(w_{h}^{j+1}\right), w_{h}^{j+1}\right) \\
& +\frac{1}{4} \rho_{\chi, h}^{2}\left(w_{h}^{n+1}\right)+\frac{1}{4} \rho_{\chi, h}^{2}\left(2 w_{h}^{n+1}-w_{h}^{n}\right)+\Delta t \sum_{j=1}^{n} \frac{\Delta t^{3}}{4} \rho_{\chi, h}^{2}\left(D_{+} D_{-} w_{h}^{j}\right)+\frac{\nu}{2} \Delta t \sum_{j=1}^{n}\left\|\nabla w_{h}^{n+1}\right\|^{2} \\
& \leq \frac{1}{4}\left\|u_{h}^{1}\right\|^{2}+\frac{1}{4}\left\|2 u_{h}^{1}-u_{h}^{0}\right\|^{2}+\frac{\Delta t}{2 \nu} \sum_{j=1}^{n}\left\|f^{j+1}\right\|_{\star}^{2} .
\end{aligned}
$$

Proof. In Step 1.1 in Algorithm 1.1 set $v_{h}=w_{h}^{n+1}$. Using the identity

$$
\frac{1}{4}\left[a^{2}+(2 a-b)^{2}\right]-\frac{1}{4}\left[b^{2}+(2 b-c)^{2}\right]+\frac{1}{4}(a-2 b+c)^{2}=\frac{1}{2}(3 a-4 b+c) a,
$$

gives

$$
\begin{aligned}
& \frac{1}{4 \Delta t}\left(\left\|u_{h}^{n+1}\right\|^{2}+\left\|2 u_{h}^{n+1}-u_{h}^{n}\right\|^{2}\right)-\frac{1}{4 \Delta t}\left(\left\|u_{h}^{n}\right\|^{2}+\left\|2 u_{h}^{n}-u_{h}^{n-1}\right\|^{2}\right)+\frac{1}{4 \Delta t}\left\|u_{h}^{n+1}-2 u_{h}^{n}+u_{h}^{n-1}\right\|^{2} \\
& \quad+\frac{3}{2 \Delta t}\left(\chi\left(w_{h}^{n+1}-G_{h}\left(w_{h}^{n+1}\right)\right), w_{h}^{n+1}\right)+\frac{1}{4 \Delta t}\left(\rho_{\chi, h}^{2}\left(w_{h}^{n+1}\right)+\rho_{\chi, h}^{2}\left(2 w_{h}^{n+1}-w_{h}^{n}\right)\right) \\
& \quad-\frac{1}{4 \Delta t}\left(\rho_{\chi, h}^{2}\left(w_{h}^{n}\right)+\rho_{\chi, h}^{2}\left(2 w_{h}^{n}-w_{h}^{n-1}\right)\right)+\frac{1}{4 \Delta t} \rho_{\chi, h}^{2}\left(w_{h}^{n+1}-2 w_{h}^{n}+w_{h}^{n-1}\right)+\nu\left\|\nabla w_{h}^{n+1}\right\|^{2} \\
& =\left(f^{n+1}, w_{h}^{n+1}\right) .
\end{aligned}
$$


Multiplying by $\Delta t$ and summing, with the assumption $w_{h}^{0}=w_{h}^{1}=0$, we obtain the energy equality

$$
\begin{aligned}
& \frac{1}{4}\left\|u_{h}^{n+1}\right\|^{2}+\frac{1}{4}\left\|2 u_{h}^{n+1}-u_{h}^{n}\right\|^{2}+\Delta t \sum_{j=1}^{n} \frac{1}{4 \Delta t}\left\|u_{h}^{j+1}-2 u_{h}^{j}+u_{h}^{j-1}\right\|^{2} \\
& +\Delta t \sum_{j=1}^{n} \frac{3 \chi}{2 \Delta t}\left(w_{h}^{n+1}-G_{h}\left(w_{h}^{n+1}\right), w_{h}^{n+1}\right)+\frac{1}{4} \rho_{\chi, h}^{2}\left(w_{h}^{n+1}\right)+\frac{1}{4} \rho_{\chi, h}^{2}\left(2 w_{h}^{n+1}-w_{h}^{n}\right) \\
& \quad+\Delta t \sum_{j=1}^{n} \frac{1}{4 \Delta t} \rho_{\chi, h}^{2}\left(w_{h}^{j+1}-2 w_{h}^{j}+w_{h}^{j-1}\right)+\nu \Delta t \sum_{j=1}^{n}\left\|\nabla w_{h}^{n+1}\right\|^{2} \\
& =\frac{1}{4}\left\|u_{h}^{1}\right\|^{2}+\frac{1}{4}\left\|2 u_{h}^{1}-u_{h}^{0}\right\|^{2}+\Delta t \sum_{j=1}^{n}\left(f^{j+1}, w_{h}^{j+1}\right) .
\end{aligned}
$$

Using the Cauchy-Schwarz-Young inequality on the right hand side and subsuming one term into the LHS proves global stability.

The energy equality of Proposition 3.3 shows that the total energy dissipation in Algorithm 1.1 has the components:

Viscous / Molecular Dissipation: $=\nu\left\|\nabla w_{h}^{n+1}\right\|^{2}$,

Numerical Dissipation from Step 1.1:= $\frac{\triangle t^{3}}{4}\left\|D_{+} D_{-} u_{h}^{n}\right\|^{2}$

Numerical Dissipation from Step 1.1: $=\frac{3 \chi}{2 \Delta t}\left(w_{h}^{n+1}-G_{h}\left(w_{h}^{n+1}\right), w_{h}^{n+1}\right)+\frac{\triangle t^{3}}{4} \rho_{\chi, h}^{2}\left(D_{+} D \_w_{h}^{n}\right) \geq 0$.

\section{Error analysis of the Algorithm 1.1}

In this section we present a detailed error analysis. We first establish computability of the procedure.

Lemma 4.1. Assume $\chi \in[0,1]$ and Assumptions 2.2, 2.3 hold. For Algorithm 1.1, $w_{h}^{n}, u_{h}^{n}$ exist at each time step.

Proof. The existence of a solution $w_{h}^{n}$ to (1.1) follows from the Leray-Schauder Principle [48]. Specifically, with $A: V_{h} \rightarrow V_{h}$, defined by $y=A(w)$

$$
(y, v):=-\frac{2 \Delta t}{3} b^{*}(w, w, v)-\frac{2 \Delta t}{3} \nu(\nabla w, \nabla v)+\frac{1}{3}\left(4 u_{h}^{n-1}-u_{h}^{n-2}, v\right)+\frac{2 \Delta t}{3}\left(f^{n}, v\right),
$$

the operator $A$ is compact and any solution of $w=s A(w)$, for $0 \leq s<1$, satisfies the bound $\|w\| \leq \gamma$, where $\gamma$ is independent of $s$.

The existence and uniqueness of $\overline{w_{h}^{n}}$ follows directly from the assumption on the well-posedness of the regularization operator. The existence and uniqueness of $u_{h}^{n}$ follows from that for $w_{h}^{n}$ and ${\overline{w_{h}^{n}}}^{h}$ and the definition of $G_{h}$.

In order to establish the optimal asymptotic error estimates for the approximation we need to assume that the true solution is more regular than that given by $(2.1),(2.2)$.

$$
\begin{aligned}
& u \in L^{\infty}\left(0, T ; W_{4}^{k+1}(\Omega)\right) \cap H^{1}\left(0, T ; H^{k+1}(\Omega)\right) \cap H^{3}\left(0, T ; L^{2}(\Omega)\right) \cap W_{4}^{2}\left(0, T ; H^{1}(\Omega)\right), \\
& p \in L^{\infty}\left(0, T ; H^{s+1}(\Omega)\right), \text { and } f \in H^{2}\left(0, T ; L^{2}(\Omega)\right) .
\end{aligned}
$$


For clarity of presentation, we introduce the mesh dependent, nonnegative (energy dissipative) homogeneous weighted functionals

$$
\rho_{\chi, 1}(v):=\left(v-\mathcal{G}_{h}\left(w_{h}^{n}\right) v, v\right)^{\frac{1}{2}}, \quad \rho_{\chi, 2}(v):=\left(v-\mathcal{G}_{h}\left(w_{h}^{n}\right) v, \mathcal{G}_{h}\left(w_{h}^{n}\right) v\right)^{\frac{1}{2}}, \quad\|v\|_{\chi, 3}:=\left(v-\mathcal{G}_{h}\left(w_{h}^{n}\right) v, v-\mathcal{G}_{h}\left(w_{h}^{n}\right) v\right)^{\frac{1}{2}}
$$

These are defined on $X_{h}$, weighted by the computed velocities $w_{h}^{n}$; the dependence on $w_{h}^{n}$ will be suppressed. For the error between $u\left(t_{n}\right)$ and $u_{h}^{n}$, and $u\left(t_{n}\right)$ and $w_{h}^{n}$, we have the following result.

Theorem 4.2. For $u, p$, and $f$ as described by (4.1), (4.2), satisfying (2.3)-(2.4), and $u_{h}^{n}$, $w_{h}^{n}$ given by Algorithm 1.1 we have that, for $\Delta t$ sufficiently small,

$$
\begin{aligned}
& \left\|\left|u-u_{h}\right|\right\|_{\infty, 0} \leq \mathcal{F}(\Delta t, h, \chi)+C h^{k+1}\||u|\|_{\infty, k+1}+C \Delta t^{2}\left\|u_{t t}\right\|_{\infty, 0}+C\left(1+\frac{\chi}{\Delta t}\right)\left(\Delta t \sum_{j=2}^{n}\left[\epsilon\left(u^{j}\right)\right]^{2}\right)^{\frac{1}{2}}, \\
& \left\|\left|u-w_{h}\right|\right\|_{\infty, 0} \leq \mathcal{F}(\Delta t, h, \chi)+C h^{k+1}\||u|\|_{\infty, k+1}+C\left(1+\frac{\chi}{\Delta t}\right)\left(\Delta t \sum_{j=2}^{n}\left[\epsilon\left(u^{j}\right)\right]^{2}\right)^{\frac{1}{2}}, \\
& \chi(1-\chi) \rho_{\chi, 1}\left(u\left(t^{n}\right)-w_{h}^{n}\right)+\chi \rho_{\chi, 2}^{2}\left(u\left(t^{n}\right)-w_{h}^{n}\right) \leq \mathcal{F}(\Delta t, h, \chi)+C\left(1+\frac{\chi}{\Delta t}\right)\left(\Delta t \sum_{j=2}^{n}\left[\epsilon\left(u^{j}\right)\right]^{2}\right)^{\frac{1}{2}} \\
& \left(\Delta t \sum_{j=2}^{n}\left(\Delta t^{3}\left\|D_{+} D_{-}\left(u^{j-1}-u_{h}^{j-1}\right)\right\|^{2}+\nu\left\|\nabla\left(u^{j}-w_{h}^{j}\right)\right\|^{2}+\frac{\chi}{\Delta t} \rho_{\chi, 1}^{2}\left(u^{j}-w_{h}^{j}\right)\right)\right)^{\frac{1}{2}} \\
& \leq \mathcal{F}(\Delta t, h, \chi)+C\left(1+\frac{\chi}{\Delta t}\right)\left(\Delta t \sum_{j=2}^{n}\left[\epsilon\left(u^{j}\right)\right]^{2}\right)^{\frac{1}{2}},
\end{aligned}
$$

for $2 \leq n \leq N_{T}$, where

$$
\begin{aligned}
& \mathcal{F}(\Delta t, h, \chi):=C\left(\left\|u^{1}-u_{h}^{1}\right\|+\left\|2\left(u^{1}-u_{h}^{1}\right)-\left(u^{0}-u_{h}^{0}\right)\right\|\right) \\
& \quad+C \nu^{-\frac{1}{2}}\left(h^{k+\frac{1}{2}}\||u|\|_{4, k+1}^{2}+h^{k+\frac{1}{2}}\||\nabla u|\|_{4,0}^{2}+h^{s+1}\||p|\|_{2, s+1}\right) \\
& \quad+C\left(h^{k+1}\left\|u_{t}\right\|_{2, k+1}+\nu^{-1} h^{k}\||u|\|_{\infty, k+1}+\nu^{\frac{1}{2}} h^{k}\||u|\|_{2, k+1}+\frac{\chi}{\Delta t} h^{k+1}\||u|\|_{2, k+1}+\Delta t^{2}\left\|u_{\mathrm{ttt}}\right\|_{2,0}\right) .
\end{aligned}
$$

Remark 4.3 (The regularization error $\epsilon(u)$ ).

1. For the nonlinear filter, the regularization error satisfies

$$
\left(\Delta t \sum_{j=2}^{n}\left[\epsilon\left(u^{j}\right)\right]^{2}\right)^{\frac{1}{2}} \leq C\left(\delta h^{k}+h^{k+1}+\delta^{2} \min \left\{\delta^{-1},\left\|\nabla \cdot\left(a\left(w_{h}\right) \nabla u\right)\right\|\right\}\right)\|\mid u\|_{2, k+1}^{2} .
$$

2. For approximate deconvolution, provided $u \in L^{\infty}\left(0, T ; H^{2 N+2}(\Omega)\right)$ for $2 N+2 \geq k+1$, under the assumption DG1, the regularization error satisfies

$$
\left(\Delta t \sum_{j=2}^{n}\left[\epsilon\left(u^{j}\right)\right]^{2}\right)^{\frac{1}{2}} \leq C\left(\delta^{2 N+2}+\delta h^{k}+h^{k+1}\right)\left(\||u|\|_{2,2 N+2}^{2}+\||u|\|_{2, k+1}^{2}\right) .
$$


Proof of Theorem 4.2. At time $t^{n}=n \Delta t, u$ given by (2.3)-(2.4) satisfies

$$
\begin{aligned}
& \left(3 u\left(t^{n}\right)-4 u\left(t^{n-1}\right)+u\left(t^{n-2}\right), v_{h}\right)+2 \Delta t \nu\left(\nabla u\left(t^{n}\right), \nabla v_{h}\right)+2 \Delta t b^{*}\left(u\left(t^{n}\right), u\left(t^{n}\right), v_{h}\right)-2 \Delta t\left(p\left(t^{n}\right), \nabla \cdot v_{h}\right) \\
& \quad=2 \Delta t\left(f\left(t^{n}\right), v_{h}\right)+\Delta t \operatorname{Intp}\left(u^{n} ; v_{h}\right),
\end{aligned}
$$

for all $v_{h} \in V_{h}$, where $\frac{1}{2} \operatorname{Intp}\left(u^{n} ; v_{h}\right)$ is the local truncation error. Subtracting (1.1) from (4.8), we have for $\varepsilon^{n}=u\left(t^{n}\right)-w_{h}^{n}$, and the pointwise error $e^{n}=u\left(t^{n}\right)-u_{h}^{n}$, (recall that $\left.f^{n}=f\left(t^{n}\right)\right)$

$$
\begin{aligned}
& \left(3 \varepsilon^{n}-4 e^{n-1}+e^{n-2}, v_{h}\right)+2 \Delta t \nu\left(\nabla \varepsilon^{n}, \nabla v_{h}\right)=-2 \Delta t b^{*}\left(\varepsilon^{n}, u\left(t^{n}\right), v_{h}\right) \\
& -2 \Delta t b^{*}\left(w_{h}^{n}, \varepsilon^{n}, v_{h}\right)+2 \Delta t\left(p\left(t^{n}\right)-p_{h}^{n}, \nabla \cdot v_{h}\right)+\Delta t \operatorname{Intp}\left(u^{n} ; v_{h}\right),
\end{aligned}
$$

for all $v_{h} \in V_{h}$.

Let $U^{n} \in V_{h}, \varepsilon^{n}=u\left(t^{n}\right)-w_{h}^{n}=\left(u\left(t^{n}\right)-U^{n}\right)+\left(U^{n}-w_{h}^{n}\right):=\Lambda^{n}+F^{n}$, and $e^{n}=u\left(t^{n}\right)-u_{h}^{n}=$ $\left(u\left(t^{n}\right)-U^{n}\right)+\left(U^{n}-u_{h}^{n}\right):=\Lambda^{n}+E^{n}$. With the choice $v_{h}=F^{n}$, using $\left(\nabla \cdot F^{n}, q_{h}\right)=0, \forall q_{h} \in Q_{h}$, and (3.2) we obtain

$$
\begin{aligned}
\frac{1}{2} & \left\|E^{n}\right\|^{2}+\frac{1}{2}\left\|2 E^{n}-E^{n-1}\right\|^{2}-\frac{1}{2}\left\|E^{n-1}\right\|^{2}-\frac{1}{2}\left\|2 E^{n-1}-E^{n-2}\right\|^{2}+\frac{1}{2}\left\|E^{n}-2 E^{n-1}+E^{n-2}\right\|^{2} \\
& +3\left(F^{n}-E^{n}, F^{n}\right)+\left(3 E^{n}-4 E^{n-1}+E^{n-2}, F^{n}-E^{n}\right)+2 \Delta t \nu\left\|\nabla F^{n}\right\|^{2} \\
= & -\left(3 \Lambda^{n}-4 \Lambda^{n-1}+\Lambda^{n-2}, F^{n}\right)-2 \Delta t \nu\left(\nabla \Lambda^{n}, \nabla F^{n}\right) \\
& -2 \Delta t b^{*}\left(\Lambda^{n}, u\left(t^{n}\right), F^{n}\right)-2 \Delta t b^{*}\left(F^{n}, u\left(t^{n}\right), F^{n}\right)-2 \Delta t b^{*}\left(w_{h}^{n}, \Lambda^{n}, F^{n}\right) \\
& +2 \Delta t\left(p\left(t^{n}\right)-p_{h}^{n}-q_{h}, \nabla \cdot F^{n}\right)+\Delta \operatorname{tn} \operatorname{Int}\left(u^{n} ; F^{n}\right) .
\end{aligned}
$$

Summing for $j=2$ to $n$ yields

$$
\begin{aligned}
& \frac{1}{2}\left\|E^{n}\right\|^{2}+\frac{1}{2}\left\|2 E^{n}-E^{n-1}\right\|^{2}+\frac{1}{2} \sum_{j=2}^{n}\left\|E^{j}-2 E^{j-1}+E^{j-2}\right\|^{2} \\
& \quad+3 \sum_{j=2}^{n}\left(F^{j}-E^{j}, F^{j}\right)+\sum_{j=2}^{n}\left(3 E^{j}-4 E^{j-1}+E^{j-2}, F^{j}-E^{j}\right)+2 \Delta t \nu \sum_{j=2}^{n}\left\|\nabla F^{j}\right\|^{2} \\
& =\frac{1}{2}\left\|E^{1}\right\|^{2}+\frac{1}{2}\left\|2 E^{1}-E^{0}\right\|^{2}-\sum_{j=2}^{n}\left(3 \Lambda^{j}-4 \Lambda^{j-1}+\Lambda^{j-2}, F^{j}\right)-2 \Delta t \nu \sum_{j=2}^{n}\left(\nabla \Lambda^{j}, \nabla F^{j}\right) \\
& \quad-2 \Delta t \sum_{j=2}^{n} b^{*}\left(\Lambda^{j}, u\left(t^{j}\right), F^{j}\right)-2 \Delta t \sum_{j=2}^{n} b^{*}\left(F^{j}, u\left(t^{j}\right), F^{j}\right)-2 \Delta t \sum_{j=2}^{n} b^{*}\left(w_{h}^{j}, \Lambda^{j}, F^{j}\right) \\
& \quad+2 \Delta t \sum_{j=2}^{n}\left(p\left(t^{j}\right)-p_{h}^{j}-q_{h}, \nabla \cdot F^{j}\right)+\Delta t \sum_{j=2}^{n} \operatorname{Intp}\left(u^{j} ; F^{j}\right) .
\end{aligned}
$$

The relations (4.4)-(4.7) will be obtained from an estimate on $F^{n}$, which will be next derived from (4.10). For reader's convenience we present this derivation in several steps.

First step. We estimate the terms on the RHS of (4.10) individually.

$$
\begin{aligned}
& \left(3 \Lambda^{j}-4 \Lambda^{j-1}+\Lambda^{j-2}, F^{j}\right)=2 \Delta t\left(\frac{3 \Lambda^{j}-4 \Lambda^{j-1}+\Lambda^{j-2}}{2 \Delta t}, F^{j}\right)=2 \Delta t\left(\Lambda_{t}\left(t^{j}\right), F^{j}\right) \leq \Delta t\left\|\Lambda_{t}\left(t^{j}\right)\right\|^{2}+\Delta t\left\|F^{j}\right\|^{2}, \\
& 2 \Delta t \nu\left(\nabla \Lambda^{j}, \nabla F^{j}\right) \leq \Delta t \nu\left\|\nabla F^{j}\right\|^{2}+\Delta t \nu\left\|\nabla \Lambda^{j}\right\|^{2} .
\end{aligned}
$$


Using $b^{*}(u, v, w) \leq C(\Omega) \sqrt{\|u\|\|\nabla u\|}\|\nabla v\|\|\nabla w\|$, for $u, v, w \in X$, and Young's inequality, we bound also the nonlinear terms

$$
\begin{aligned}
& b^{*}\left(\Lambda^{j}, u\left(t^{j}\right), F^{j}\right) \leq C \sqrt{\left\|\Lambda^{j}\right\|\left\|\nabla \Lambda^{j}\right\|}\left\|\nabla u\left(t^{j}\right)\right\|\left\|\nabla F^{j}\right\| \leq \frac{\nu}{16}\left\|\nabla F^{j}\right\|^{2}+C \nu^{-1}\left\|\Lambda^{j}\right\|\left\|\nabla \Lambda^{j}\right\|\left\|\nabla u\left(t^{j}\right)\right\|^{2}, \\
& b^{*}\left(F^{j}, u\left(t^{j}\right), F^{j}\right) \leq C\left\|F^{j}\right\|^{1 / 2}\left\|\nabla F^{j}\right\|^{3 / 2}\left\|\nabla u\left(t^{j}\right)\right\| \leq \frac{\nu}{16}\left\|\nabla F^{j}\right\|^{2}+C \nu^{-3}\left\|\nabla u\left(t^{j}\right)\right\|^{4}\left\|F^{j}\right\|^{2}, \\
& b^{*}\left(w_{h}^{j}, \Lambda^{j}, F^{j}\right) \leq C\left\|\nabla w_{h}^{j}\right\|\left\|\nabla \Lambda^{j}\right\|\left\|\nabla F^{j}\right\| \leq \frac{\nu}{16}\left\|\nabla F^{j}\right\|^{2}+C \nu^{-1}\left\|\nabla w_{h}^{j}\right\|^{2}\left\|\nabla \Lambda^{j}\right\|^{2}, \\
& \left(p\left(t^{j}\right)-p_{h}^{j}-q_{h}, \nabla \cdot F^{j}\right) \leq\left\|p\left(t^{j}\right)-p_{h}^{j}-q_{h}\right\|\left\|\nabla \cdot F^{j}\right\| \leq \frac{\nu}{16}\left\|\nabla F^{j}\right\|^{2}+C \nu^{-1}\left\|p\left(t^{j}\right)-p_{h}^{j}-q_{h}\right\|^{2} .
\end{aligned}
$$

With the bounds (4.11)-(4.16), the relation (4.10) becomes

$$
\begin{aligned}
& \frac{1}{2}\left\|E^{n}\right\|^{2}+\frac{1}{2}\left\|2 E^{n}-E^{n-1}\right\|^{2}+\frac{1}{2} \sum_{j=2}^{n}\left\|E^{j}-2 E^{j-1}+E^{j-2}\right\|^{2} \\
& \quad+3 \sum_{j=2}^{n}\left(F^{j}-E^{j}, F^{j}\right)+\sum_{j=2}^{n}\left(3 E^{j}-4 E^{j-1}+E^{j-2}, F^{j}-E^{j}\right)+\frac{\Delta t \nu}{2} \sum_{j=2}^{n}\left\|\nabla F^{j}\right\|^{2} \\
& \leq \frac{1}{2}\left\|E^{1}\right\|^{2}+\frac{1}{2}\left\|2 E^{1}-E^{0}\right\|^{2}+\Delta t \sum_{j=2}^{n}\left\|\Lambda_{t}\left(t^{j}\right)\right\|^{2}+C \Delta t \sum_{j=2}^{n}\left(1+\nu^{-3}\left\|\nabla u\left(t^{j}\right)\right\|^{4}\right)\left\|F^{j}\right\|^{2}+\Delta t \nu \sum_{j=2}^{n}\left\|\nabla \Lambda^{j}\right\|^{2} \\
& \quad+C \nu^{-1} \Delta t \sum_{j=2}^{n}\left\|\Lambda^{j}\right\|\left\|\nabla \Lambda^{j}\right\|\left\|\nabla u^{j}\right\|^{2}+C \nu^{-1} \Delta t \sum_{j=2}^{n}\left\|\nabla w_{h}^{j}\right\|^{2}\left\|\nabla \Lambda^{j}\right\|^{2} \\
& \quad+2 C \nu^{-1} \Delta t \sum_{j=2}^{n}\left\|p\left(t^{j}\right)-p_{h}^{j}-q_{h}\right\|^{2}+\Delta t \sum_{j=2}^{n} \operatorname{Intp}\left(u^{j} ; F^{j}\right) .
\end{aligned}
$$

Second step. We now estimate the LHS of (4.10) and express it in terms of $F^{n}$. As $u_{h}^{n}$ and $w_{h}^{n}$ are connected through Step 1.1 of Algorithm 1.1, we use equation (1.2) to obtain a relationship between $\left\|F^{n}\right\|$ and $\left\|E^{n}\right\|$. The true solution $u\left(\cdot, t^{n}\right)=u^{n}$ satisfies

$$
u^{n}=(1-\chi) u^{n}+\chi \mathcal{G}_{h}\left(w_{h}^{n}\right) u^{n}+\chi\left(u^{n}-\mathcal{G}_{h}\left(w_{h}^{n}\right) u^{n}\right) .
$$

Subtracting (1.2) evaluated at $n$ from (4.18) yields

$$
e^{n}=(1-\chi) \varepsilon^{n}+\chi \mathcal{G}_{h}\left(w_{h}^{n}\right) \varepsilon^{n}+\chi\left(u^{n}-\mathcal{G}_{h}\left(w_{h}^{n}\right) u^{n}\right),
$$

and equivalently

$$
E^{n}=(1-\chi) F^{n}+\chi \mathcal{G}_{h}\left(w_{h}^{n}\right) F^{n}-\chi\left(I-\mathcal{G}_{h}\left(w_{h}^{n}\right)\right) \Lambda^{n}+\chi\left(I-\mathcal{G}_{h}\left(w_{h}^{n}\right)\right) u^{n} .
$$

Using the assumption (1.3) $(\chi \in[0,1])$ and (2.10), this implies

$$
\left\|E^{n}\right\| \leq\left\|F^{n}\right\|+\chi\left\|\left(I-\mathcal{G}_{h}\left(w_{h}^{n}\right)\right) \Lambda^{n}\right\|+\chi\left\|\left(I-\mathcal{G}_{h}\left(w_{h}^{n}\right)\right) u^{n}\right\|,
$$

and squaring up and simplifying

$$
\left\|E^{n}\right\|^{2} \leq\left\|F^{n}\right\|^{2}+\Delta t\left\|F^{n}\right\|^{2}+2 \chi^{2}\left(1+(\Delta t)^{-1}\right)\left\|\Lambda^{n}-\mathcal{G}_{h}\left(w_{h}^{n}\right) \Lambda^{n}\right\|^{2}+2 \chi^{2}\left(1+(\Delta t)^{-1}\right)\left\|u^{n}-\mathcal{G}_{h}\left(w_{h}^{n}\right) u^{n}\right\|^{2} .
$$

Note that by Cauchy-Schwarz inequality we have

$$
\frac{1}{2}\left\|F^{n}\right\|^{2}+\left(\left\|F^{n}\right\|^{2}-\left\|E^{n}\right\|^{2}\right) \leq \frac{1}{2}\left\|E^{n}\right\|^{2}+3\left(F^{n}-E^{n}, F^{n}\right)
$$


Third step. Using the bound (4.22) into (4.17) yields

$$
\begin{aligned}
& \frac{1}{2}\left\|F^{n}\right\|^{2}+\frac{1}{2}\left\|2 E^{n}-E^{n-1}\right\|^{2}+\frac{1}{2} \sum_{j=2}^{n}\left\|E^{j}-2 E^{j-1}+E^{j-2}\right\|^{2} \\
& \quad+3 \sum_{j=2}^{n-1}\left(F^{j}-E^{j}, F^{j}\right)+\sum_{j=2}^{n}\left(3 E^{j}-4 E^{j-1}+E^{j-2}, F^{j}-E^{j}\right)+\frac{\Delta t \nu}{2} \sum_{j=2}^{n}\left\|\nabla F^{j}\right\|^{2} \\
& \leq \Delta t\left\|F^{n}\right\|^{2}+2 \chi^{2}\left(1+(\Delta t)^{-1}\right)\left\|\Lambda^{n}-\mathcal{G}_{h}\left(w_{h}^{n}\right) \Lambda^{n}\right\|^{2}+2 \chi^{2}\left(1+(\Delta t)^{-1}\right)\left\|u^{n}-\mathcal{G}_{h}\left(w_{h}^{n}\right) u^{n}\right\|^{2} \\
& \quad+\frac{1}{2}\left\|E^{1}\right\|^{2}+\frac{1}{2}\left\|2 E^{1}-E^{0}\right\|^{2}+\Delta t \sum_{j=2}^{n}\left\|\Lambda_{t}\left(t^{j}\right)\right\|^{2}+C \Delta t \sum_{j=2}^{n}\left(1+\nu^{-3}\left\|\nabla u\left(t^{j}\right)\right\|^{4}\right)\left\|F^{j}\right\|^{2}+\Delta t \nu \sum_{j=2}^{n}\left\|\nabla \Lambda^{j}\right\|^{2} \\
& \quad+2 C \nu^{-1} \Delta t \sum_{j=2}^{n}\left\|\Lambda^{j}\right\|\left\|\nabla \Lambda^{j}\right\|\left\|\nabla u^{j}\right\|^{2}+2 C \nu^{-1} \Delta t \sum_{j=2}^{n}\left\|\nabla w_{h}^{j}\right\|^{2}\left\|\nabla \Lambda^{j}\right\|^{2} \\
& \quad+2 C \nu^{-1} \Delta t \sum_{j=2}^{n}\left\|p\left(t^{j}\right)-p_{h}^{j}-q_{h}\right\|^{2}+\Delta t \sum_{j=2}^{n} \operatorname{Intp}\left(u^{j} ; F^{j}\right) .
\end{aligned}
$$

Fourth step. In order to further simplify the LHS of (4.23), note that (4.20) also yields

$$
\begin{aligned}
F^{j}-E^{j}= & \chi\left(I-\mathcal{G}_{h}\left(w_{h}^{n}\right)\right) F^{j}+\chi\left(I-\mathcal{G}_{h}\left(w_{h}^{n}\right)\right) \Lambda^{j}-\chi\left(I-\mathcal{G}_{h}\left(w_{h}^{n}\right)\right) u^{j}, \\
3 E^{j}-4 E^{j-1}+E^{j-2}= & (1-\chi)\left(3 F^{j}-4 F^{j-1}+F^{j-2}\right)+\chi \mathcal{G}_{h}\left(w_{h}^{n}\right)\left(3 F^{j}-4 F^{j-1}+F^{j-2}\right) \\
& -\chi\left(I-\mathcal{G}_{h}\left(w_{h}^{n}\right)\right)\left(3 \Lambda^{j}-4 \Lambda^{j-1}+\Lambda^{j-2}\right)+\chi\left(I-\mathcal{G}_{h}\left(w_{h}^{n}\right)\right)\left(3 u^{j}-4 u^{j-1}+u^{j-2}\right) .
\end{aligned}
$$

Then using the identity (3.2), Cauchy-Schwarz, Assumptions (2.8)-(2.9) $\left(\left\|\mathcal{G}_{h}\left(w_{h}^{j}\right)\right\| \leq 1,\left\|I-\mathcal{G}_{h}\left(w_{h}^{j}\right)\right\| \leq 1\right)$, the assumption $\left\|F^{1}\right\|=\left\|2 F^{1}-F^{0}\right\|=0$, the Minkowski inequality yields the following lower bound for the fourth and fifth terms in (4.23)

$$
\begin{aligned}
& 3 \sum_{j=2}^{n-1}\left(F^{j}-E^{j}, F^{j}\right)+\sum_{j=2}^{n}\left(3 E^{j}-4 E^{j-1}+E^{j-2}, F^{j}-E^{j}\right) \\
& \geq \mathcal{C}_{1, \chi, T}-\frac{\chi^{2}}{2}\left\|\Lambda^{n}\right\|_{\chi, 3}^{2}-\frac{1}{2} \chi^{2}\left\|2 \Lambda^{n}-\Lambda^{n-1}\right\|_{\chi, 3}^{2}-\frac{\chi^{2}}{2}\left\|u^{n}\right\|_{\chi, 3}^{2}-\frac{1}{2} \chi^{2}\left\|2 u^{n}-u^{n-1}\right\|_{\chi, 3}^{2}-\frac{\chi^{2}}{2}\left\|u^{n}-2 u^{n-1}+u^{n-2}\right\|_{\chi, 3} \\
& \quad-\Delta t \sum_{j=0}^{n}\left\|F^{j}\right\|^{2}-C \frac{\chi^{2}}{\Delta t}(1+\chi) \sum_{j=2}^{n}\left\|\left(I-\mathcal{G}_{h}\left(w_{h}^{j}\right)\right) \Lambda^{j}\right\|^{2} \\
& \quad-C \Delta t\left(1+\chi+\frac{\chi^{2}}{(\Delta t)^{2}}+\frac{\chi^{3}}{(\Delta t)^{2}}+\frac{\chi^{4}}{(\Delta t)^{2}}\right) \sum_{j=2}^{n}\left\|\left(I-\mathcal{G}_{h}\left(w_{h}^{j}\right)\right) u^{j}\right\|^{2}
\end{aligned}
$$

where

$$
\mathcal{C}_{1, \chi, T}=3 \chi \sum_{j=2}^{n-1} \rho_{\chi, 1}^{2}\left(F^{j}\right)+\frac{(1-\chi) \chi}{2} \rho_{\chi, 1}^{2}\left(F^{n}\right)+\frac{\chi^{2}}{2} \rho_{\chi, 2}^{2}\left(F^{n}\right)
$$


Fifth step. Using (4.24) into (4.23) we obtain (use $\chi \in[0,1], \Delta t<1,(2.7),(2.10)$ )

$$
\begin{aligned}
& \frac{1}{2}\left\|F^{n}\right\|^{2}+\mathcal{C}_{T}+\frac{\Delta t \nu}{2} \sum_{j=2}^{n}\left\|\nabla F^{j}\right\|^{2} \\
& \leq \mathcal{C}_{0}+C \chi^{2}(\Delta t)^{-1} \sum_{j=2}^{n}\left\|\Lambda^{j}\right\|^{2}+C\left(1+\chi^{2}(\Delta t)^{-2}\right) \Delta t \sum_{j=2}^{n}\left[\epsilon\left(u^{j}\right)\right]^{2} \\
& \quad+\Delta t \sum_{j=2}^{n}\left\|\Lambda_{t}\left(t^{j}\right)\right\|^{2}+C \Delta t \sum_{j=0}^{n}\left(1+\nu^{-3}\left\|\nabla u\left(t^{j}\right)\right\|^{4}\right)\left\|F^{j}\right\|^{2}+\Delta t \nu \sum_{j=2}^{n}\left\|\nabla \Lambda^{j}\right\|^{2} \\
& \quad+C \nu^{-1} \Delta t \sum_{j=2}^{n}\left\|\Lambda^{j}\right\|\left\|\nabla \Lambda^{j}\right\|\left\|\nabla u^{j}\right\|^{2}+C \nu^{-1} \Delta t \sum_{j=2}^{n}\left\|\nabla w_{h}^{j}\right\|^{2}\left\|\nabla \Lambda^{j}\right\|^{2} \\
& \quad+C \nu^{-1} \Delta t \sum_{j=2}^{n}\left\|p\left(t^{j}\right)-p_{h}^{j}-q_{h}\right\|^{2}+\Delta t \sum_{j=2}^{n} \operatorname{Intp}\left(u^{j} ; F^{j}\right),
\end{aligned}
$$

where

$$
\begin{aligned}
& \mathcal{C}_{0}=\frac{1}{2}\left\|E^{1}\right\|^{2}+\frac{1}{2}\left\|2 E^{1}-E^{0}\right\|^{2} \\
& \mathcal{C}_{T}=3 \chi \sum_{j=2}^{n-1} \rho_{\chi, 1}^{2}\left(F^{j}\right)+\frac{(1-\chi) \chi}{2} \rho_{\chi, 1}^{2}\left(F^{n}\right)+\frac{\chi^{2}}{2} \rho_{\chi, 2}^{2}\left(F^{n}\right)+\frac{1}{2}\left\|2 E^{n}-E^{n-1}\right\|^{2}+\frac{1}{2} \sum_{j=2}^{n}\left\|E^{j}-2 E^{j-1}+E^{j-2}\right\|^{2} .
\end{aligned}
$$

Sixth step. Finally, we further simplify the terms in the RHS of (4.25) as follows.

$$
\begin{aligned}
& C \frac{\chi^{2}}{\Delta t} \sum_{j=2}^{n}\left\|\Lambda^{j}\right\|^{2} \leq C \frac{\chi^{2}}{\Delta t} \sum_{j=2}^{n} h^{2 k+2}\left|u^{j}\right|_{k+1}^{2} \leq C \chi^{2}(\Delta t)^{-2} h^{2 k+2}\||u|\|_{2, k+1}^{2}, \\
& \nu \Delta t \sum_{j=2}^{n}\left\|\nabla \Lambda^{j}\right\|^{2} \leq C \nu \Delta t \sum_{j=2}^{n} h^{2 k}\left|u^{j}\right|_{k+1}^{2} \leq C \nu h^{2 k}\||u|\|_{2, k+1}^{2} .
\end{aligned}
$$

For the next term

$$
\begin{aligned}
& C \nu^{-1} \Delta t \sum_{j=2}^{n}\left\|\nabla u^{j}\right\|^{2}\left\|\Lambda^{j}\right\|\left\|\nabla \Lambda^{j}\right\| \leq C \nu^{-1} h^{2 k+1} \Delta t \sum_{j=2}^{n}\left|u^{j}\right|_{k+1}^{2}\left\|\nabla u^{j}\right\|^{2} \\
& \leq C \nu^{-1} h^{2 k+1} \Delta t\left(\sum_{j=2}^{n}\left|u^{j}\right|_{k+1}^{4}+\sum_{j=2}^{n}\left\|\nabla u^{j}\right\|^{4}\right) \leq C \nu^{-1} h^{2 k+1}\left(\||u|\|_{4, k+1}^{4}+\||\nabla u|\|_{4,0}^{4}\right) .
\end{aligned}
$$

Using the boundedness of $\nu \Delta t \sum_{j=2}^{n}\left\|\nabla w_{h}^{j}\right\|$ (Prop. 3.3)

$$
C \nu^{-1} \Delta t \sum_{j=2}^{n}\left\|\nabla w_{h}^{j}\right\|^{2}\left\|\nabla \Lambda^{j}\right\|^{2} \leq C \nu^{-2} h^{2 k}\|\mid u\|_{\infty, k+1}^{2} .
$$

Next

$$
\begin{aligned}
& \Delta t \sum_{j=2}^{n}\left\|\Lambda_{t}\left(t^{j}\right)\right\|^{2} \leq \Delta t \sum_{j=2}^{n} h^{2 k+2}\left\|u_{t}\left(t^{j}\right)\right\|_{k+1}^{2} \leq h^{2 k+2}\left\|u_{t}\right\|_{2, k+1}^{2} . \\
& \nu^{-1} \Delta t \sum_{j=2}^{n}\left\|p\left(t^{j}\right)-p_{h}^{j}-q_{h}\right\|^{2} \leq \nu^{-1} \Delta t \sum_{n=1}^{l} h^{2 s+2}\left\|p\left(t^{j}\right)\right\|_{s+1}^{2} \leq C \nu^{-1} h^{2 s+2}\||p|\|_{2, s+1}^{2} .
\end{aligned}
$$


As in [20] the interpolation error in (4.25) can be bounded as

$$
\Delta t \sum_{j=2}^{n}\left|\operatorname{Intp}\left(u^{n} ; F^{n}\right)\right| \leq \Delta t \sum_{j=2}^{n}\left\|F^{j}\right\|^{2}+C(\Delta t)^{4}\left\|u_{\mathrm{ttt}}\right\|_{2,0}^{2} .
$$

Seventh step. In conclusion, combining (4.27)-(4.31), the inequality (4.25) yields the desired estimate on $F^{n}$

$$
\begin{aligned}
& \frac{1}{2}\left\|F^{n}\right\|^{2}+\mathcal{C}_{T}+\frac{\Delta t \nu}{2} \sum_{j=2}^{n}\left\|\nabla F^{j}\right\|^{2} \\
& \leq \mathcal{C}_{0}+C \Delta t \sum_{j=2}^{n}\left(1+\nu^{-3}\left\|\nabla u\left(t^{j}\right)\right\|^{4}\right)\left\|F^{j}\right\|^{2}+h^{2 k+2}\left\|u_{t}\right\|_{2, k+1}^{2}+C \nu h^{2 k}\||u|\|_{2, k+1}^{2} \\
& \quad+C \nu^{-1} h^{2 k+1}\left(\||u|\|_{4, k+1}^{4}+\||\nabla u|\|_{4,0}^{4}\right)+C \nu^{-2} h^{2 k}\||u|\|_{\infty, k+1}^{2} \\
& \quad+C \nu^{-1} h^{2 s+2}\||p|\|_{2, s+1}^{2}+C \chi^{2}(\Delta t)^{-2} h^{2 k+2}\||u|\|_{2, k+1}^{2} \\
& \quad+C\left(1+\chi^{2}(\Delta t)^{-2}\right) \Delta t \sum_{j=2}^{n}\left[\epsilon\left(u^{j}\right)\right]^{2}+C(\Delta t)^{4}\left\|u_{\mathrm{ttt}}\right\|_{2,0}^{2} .
\end{aligned}
$$

The proof of Theorem 4.2 resumes with the application of the discrete Gronwall Lemma [23]. Hence, with $\Delta t$ sufficiently small, i.e. $\Delta t<C\left(1+\nu^{-3}\||\nabla u|\|_{\infty, 0}^{4}\right)^{-1}$, we have

$$
\begin{aligned}
& \left\|F^{n}\right\|^{2}+(1-\chi) \chi \rho_{\chi, 1}^{2}\left(F^{n}\right)+\chi^{2} \rho_{\chi, 2}^{2}\left(F^{n}\right)+\frac{\chi}{\Delta t} \Delta t \sum_{j=2}^{n} \rho_{\chi, 1}^{2}\left(F^{j}\right) \\
& \quad+\left\|2 E^{n}-E^{n-1}\right\|^{2}+\Delta t \sum_{j=2}^{n} \Delta t^{3}\left\|D_{+} D_{-} E^{j-1}\right\|^{2}+\Delta t \nu \sum_{j=2}^{n}\left\|\nabla F^{j}\right\|^{2} \\
& \leq C\left(\left\|E^{1}\right\|^{2}+\left\|2 E^{1}-E^{0}\right\|^{2}\right) \\
& \quad+C h^{2 k+2}\left\|u_{t}\right\|_{2, k+1}^{2}+C \nu h^{2 k}\||u|\|_{2, k+1}^{2}+C \nu^{-1} h^{2 k+1}\left(\||u|\|_{4, k+1}^{4}+\||\nabla u|\|_{4,0}^{4}\right) \\
& \quad+C \nu^{-2} h^{2 k}\||u|\|_{\infty, k+1}^{2}+C \nu^{-1} h^{2 s+2}\||p|\|_{2, s+1}^{2}+C \chi^{2}(\Delta t)^{-2} h^{2 k+2}\||u|\|_{2, k+1}^{2} \\
& \quad+C\left(1+\chi^{2}(\Delta t)^{-2}\right) \Delta t \sum_{j=2}^{n}\left[\epsilon\left(u^{j}\right)\right]^{2}+C(\Delta t)^{4}\left\|u_{\mathrm{ttt}}\right\|_{2,0}^{2} .
\end{aligned}
$$

Using the second and the third term, respectively the fourth, sixth and seventh terms in the left hand side of (4.33), we immediately obtain (4.6) and (4.7). The estimate given in (4.5) for $\left\|\left|u-w_{h}\right|\right\|_{\infty, 0}$ follows from the triangle inequality, Lemma 2.9 and (4.33). Finally, the estimate for $\left\|\left|u-u_{h}\right|\right\|_{\infty, 0}$ follows from (4.21), the triangle inequality and the estimate on $\left\|\left|u-w_{h}\right|\right\|_{\infty, 0}$.

For the case of Taylor-Hood approximating elements, i.e. $k=2, s=1$, we have the following asymptotic estimate. 
Corollary 4.4. Under the assumptions of Theorem 4.2, with $\delta=C h$ and $\left(X_{h}, Q_{h}\right)$ given by the Taylor-Hood approximation elements, we have

$$
\begin{aligned}
\left\|\left|u-w_{h}\right|\right\|_{\infty, 0}+\left\|\left|u-u_{h}\right|\right\|_{\infty, 0}+ & {\left[\Delta t \sum_{j=2}^{n}\left(\Delta t^{3}\left\|D_{+} D_{-}\left(u^{n}-u_{h}^{n}\right)\right\|^{2}+\nu\left\|\nabla\left(u^{n}-w_{h}^{n}\right)\right\|^{2}+\frac{\chi}{\Delta t} \rho_{\chi, 1}^{2}\left(u^{n}-w_{h}^{n}\right)\right)\right]^{1 / 2} } \\
\leq & C(u, p, \nu)\left\{\Delta t^{2}+h^{2}+\frac{h^{3}}{\Delta t}+\frac{\chi}{\Delta t}\left(\Delta t \sum_{j=2}^{n}\left[\epsilon\left(u^{j}\right)\right]^{2}\right)^{\frac{1}{2}}\right\}
\end{aligned}
$$

Next we analyze the effect of Step 1.1 on stability and accuracy, phrased in terms of the functional $\rho_{\chi, 1}$, defined in (4.3). This functional has the property of controlling small scale, fluctuating components of the error. Separating $u_{h}$ and $w_{h}$, estimate (4.35) shows that the high frequency components of $w_{h}^{n}$, typically spurious oscillations, are diminished in forming $u_{h}^{n}$, i.e., $\left\|u_{h}^{n}\right\|_{\chi, 1}<\rho_{\chi, 1}\left(w_{h}^{n}\right)$. The second estimate (4.36) establishes a relationship between the high frequency components of the error in $u_{h}^{n}$ and $w_{h}^{n}$, i.e. $\rho_{\chi, 1}\left(u^{n}-u_{h}^{n}\right)$ and $\rho_{\chi, 1}\left(u^{n}-w_{h}^{n}\right)$, respectively. Note that taking $\phi:=\left(I-\mathcal{G}_{h}\left(w_{h}^{n}\right)\right) v$ in assumptions $(2.8)-(2.10)$ we obtain

$$
\left(\left(I-\mathcal{G}_{h}\left(w_{h}^{n}\right)\right) v,\left(I-\mathcal{G}_{h}\left(w_{h}^{n}\right)\right) \mathcal{G}_{h}\left(w_{h}^{n}\right) v\right) \geq 0 \quad \forall v \in V_{h} .
$$

Theorem 4.5. Under the assumptions of Theorem 4.2, for $n=1,2, \ldots, N_{T}, 0 \leq \delta \leq 1$,

$$
\begin{gathered}
\rho_{\chi, 1}^{2}\left(u_{h}^{n}\right)=\rho_{\chi, 1}^{2}\left(w_{h}^{n}\right)-\chi(2-\chi)\left\|w_{h}^{n}\right\|_{\chi, 3}^{2}-\chi^{2}\left(w_{h}^{n}-\mathcal{G}_{h}\left(w_{h}^{n}\right) w_{h}^{n},\left(I-\mathcal{G}_{h}\left(w_{h}^{n}\right)\right) \mathcal{G}_{h}\left(w_{h}^{n}\right) w_{h}^{n}\right), \\
\rho_{\chi, 1}^{2}\left(u^{n}-u_{h}^{n}\right) \leq \rho_{\chi, 1}^{2}\left(u^{n}-w_{h}^{n}\right)-\chi^{2}\left(\left(I-\mathcal{G}_{h}\left(w_{h}^{n}\right)\right)\left(u^{n}-w_{h}^{n}\right),\left(I-\mathcal{G}_{h}\left(w_{h}^{n}\right)\right) \mathcal{G}_{h}\left(w_{h}^{n}\right)\left(u^{n}-w_{h}^{n}\right)\right) \\
-\frac{3}{2} \chi(1-\chi)\left\|u^{n}-w_{h}^{n}\right\|_{\chi, 3}^{2}+2 \chi(1+\chi)\left\|u^{n}\right\|_{\chi, 3}^{2}+\chi^{2} \rho_{\chi, 1}^{2}\left(\left(I-\mathcal{G}_{h}\left(w_{h}^{n}\right)\right) u^{n}\right) .
\end{gathered}
$$

Proof. Taking the inner product of both sides of (1.2) at level $n$ with $u_{h}^{n}-\mathcal{G}_{h}\left(w_{h}^{n}\right) u_{h}^{n}$, we obtain

$$
\begin{aligned}
\rho_{\chi, 1}^{2}\left(u_{h}^{n}\right)=\left(u_{h}^{n},\left(I-\mathcal{G}_{h}\left(w_{h}^{n}\right)\right) u_{h}^{n}\right) \\
=\left(w_{h}^{n}-\chi\left(I-\mathcal{G}_{h}\left(w_{h}^{n}\right)\right) w_{h}^{n},\left(I-\mathcal{G}_{h}\left(w_{h}^{n}\right)\right)\left[w_{h}^{n}-\chi\left(I-D_{h} G_{h}\right) w_{h}^{n}\right]\right) \\
=\rho_{\chi, 1}^{2}\left(w_{h}^{n}\right)+\chi^{2}\left(\left(I-\mathcal{G}_{h}\left(w_{h}^{n}\right)\right) w_{h}^{n},\left(I-\mathcal{G}_{h}\left(w_{h}^{n}\right)\right)^{2} w_{h}^{n}\right)-\chi\left(w_{h}^{n},\left(I-\mathcal{G}_{h}\left(w_{h}^{n}\right)\right)^{2} w_{h}^{n}\right) \\
\quad-\chi\left(\left(I-\mathcal{G}_{h}\left(w_{h}^{n}\right)\right) w_{h}^{n},\left(I-\mathcal{G}_{h}\left(w_{h}^{n}\right)\right) w_{h}^{n}\right) \\
=\rho_{\chi, 1}^{2}\left(w_{h}^{n}\right)+\chi^{2}\left\|w_{h}^{n}\right\|_{\chi, 3}^{2}-\chi^{2}\left(\left(I-\mathcal{G}_{h}\left(w_{h}^{n}\right)\right) w_{h}^{n},\left(I-\mathcal{G}_{h}\left(w_{h}^{n}\right)\right) \mathcal{G}_{h}\left(w_{h}^{n}\right) w_{h}^{n}\right)-2 \chi\left\|w_{h}^{n}\right\|_{\chi, 3}^{2} \\
=\rho_{\chi, 1}^{2}\left(w_{h}^{n}\right)-\chi(2-\chi)\left\|w_{h}^{n}\right\|_{\chi, 3}^{2}-\chi^{2}\left(\left(I-\mathcal{G}_{h}\left(w_{h}^{n}\right)\right) w_{h}^{n},\left(I-\mathcal{G}_{h}\left(w_{h}^{n}\right)\right) \mathcal{G}_{h}\left(w_{h}^{n}\right) w_{h}^{n}\right),
\end{aligned}
$$

which establishes (4.35).

To establish (4.36) we begin with (4.19). Taking the inner product of both sides with $\varepsilon^{n}-\mathcal{G}_{h}\left(w_{h}^{n}\right) \varepsilon^{n}$,

$$
\left(e^{n},\left(I-\mathcal{G}_{h}\left(w_{h}^{n}\right)\right) \varepsilon^{n}\right)=\rho_{\chi, 1}^{2}\left(\varepsilon^{n}\right)-\chi\left\|\varepsilon^{n}\right\|_{\chi, 3}^{2}+\chi\left(\left(I-\mathcal{G}_{h}\left(w_{h}^{n}\right)\right) u^{n},\left(I-\mathcal{G}_{h}\left(w_{h}^{n}\right)\right) \varepsilon^{n}\right),
$$

and by the polarization identity

$$
\frac{1}{2} \rho_{\chi, 1}^{2}\left(e^{n}\right)+\frac{1}{2} \rho_{\chi, 1}^{2}\left(\varepsilon^{n}\right)-\frac{1}{2} \rho_{\chi, 1}^{2}\left(e^{n}-\varepsilon^{n}\right)=\rho_{\chi, 1}^{2}\left(\varepsilon^{n}\right)-\chi\left\|\varepsilon^{n}\right\|_{\chi, 3}^{2}+\chi\left(\left(I-\mathcal{G}_{h}\left(w_{h}^{n}\right)\right) u^{n},\left(I-\mathcal{G}_{h}\left(w_{h}^{n}\right)\right) \varepsilon^{n}\right) .
$$

Thus,

$$
\rho_{\chi, 1}^{2}\left(\varepsilon^{n}\right)=\rho_{\chi, 1}^{2}\left(e^{n}\right)-\rho_{\chi, 1}^{2}\left(e^{n}-\varepsilon^{n}\right)+2 \chi\left\|\varepsilon^{n}\right\|_{\chi, 3}^{2}-2 \chi\left(\left(I-\mathcal{G}_{h}\left(w_{h}^{n}\right)\right) u^{n},\left(I-\mathcal{G}_{h}\left(w_{h}^{n}\right)\right) \varepsilon^{n}\right) .
$$

In addition, rearranging (4.20) we have

$$
e^{n}-\varepsilon^{n}=-\chi\left(I-\mathcal{G}_{h}\left(w_{h}^{n}\right)\right) \varepsilon^{n}+\chi\left(I-\mathcal{G}_{h}\left(w_{h}^{n}\right)\right) u^{n}
$$


and thus,

$$
\begin{aligned}
& \rho_{\chi, 1}^{2}\left(e^{n}-\varepsilon^{n}\right)=\left(\left(e^{n}-\varepsilon^{n}\right),\left(I-\mathcal{G}_{h}\left(w_{h}^{n}\right)\right)\left(e^{n}-\varepsilon^{n}\right)\right) \\
& =\chi^{2} \rho_{\chi, 1}^{2}\left(\left(I-\mathcal{G}_{h}\left(w_{h}^{n}\right)\right) \varepsilon^{n}\right)+\chi^{2} \rho_{\chi, 1}^{2}\left(\left(I-\mathcal{G}_{h}\left(w_{h}^{n}\right)\right) u^{n}\right) \\
& \quad-\chi^{2}\left(\left(I-\mathcal{G}_{h}\left(w_{h}^{n}\right)\right) \varepsilon^{n},\left(I-\mathcal{G}_{h}\left(w_{h}^{n}\right)\right)^{2} u^{n}\right)-\chi^{2}\left(\left(I-\mathcal{G}_{h}\left(w_{h}^{n}\right)\right) u^{n},\left(I-\mathcal{G}_{h}\left(w_{h}^{n}\right)\right)^{2} \varepsilon^{n}\right) .
\end{aligned}
$$

Substituting (4.38) into (4.37) and rearranging

$$
\begin{aligned}
\rho_{\chi, 1}^{2}\left(\varepsilon^{n}\right)= & \rho_{\chi, 1}^{2}\left(e^{n}\right)-\chi^{2} \rho_{\chi, 1}^{2}\left(\left(I-\mathcal{G}_{h}\left(w_{h}^{n}\right)\right) \varepsilon^{n}\right)-\chi^{2} \rho_{\chi, 1}^{2}\left(\left(I-\mathcal{G}_{h}\left(w_{h}^{n}\right)\right) u^{n}\right) \\
& +\chi^{2}\left(\left(I-\mathcal{G}_{h}\left(w_{h}^{n}\right)\right) \varepsilon^{n},\left(I-\mathcal{G}_{h}\left(w_{h}^{n}\right)\right)^{2} u^{n}\right)+\chi^{2}\left(\left(I-\mathcal{G}_{h}\left(w_{h}^{n}\right)\right) u^{n},\left(I-\mathcal{G}_{h}\left(w_{h}^{n}\right)\right)^{2} \varepsilon^{n}\right) \\
& +2 \chi\left\|\varepsilon^{n}\right\|_{\chi, 3}^{2}-2 \chi\left(\left(I-\mathcal{G}_{h}\left(w_{h}^{n}\right)\right) u^{n},\left(I-\mathcal{G}_{h}\left(w_{h}^{n}\right)\right) \varepsilon^{n}\right) .
\end{aligned}
$$

Note that

$$
\begin{gathered}
-\chi^{2} \rho_{\chi, 1}^{2}\left(\left(I-\mathcal{G}_{h}\left(w_{h}^{n}\right)\right) \varepsilon^{n}\right)+2 \chi\left\|\varepsilon^{n}\right\|_{\chi, 3}^{2}=\left(2 \chi-\chi^{2}\right)\left\|\varepsilon^{n}\right\|_{\chi, 3}^{2}+\chi^{2}\left(\left(I-\mathcal{G}_{h}\left(w_{h}^{n}\right)\right) \varepsilon^{n}, \mathcal{G}_{h}\left(w_{h}^{n}\right)\left(I-\mathcal{G}_{h}\left(w_{h}^{n}\right)\right) \varepsilon^{n}\right) \\
\chi^{2}\left(\left(I-\mathcal{G}_{h}\left(w_{h}^{n}\right)\right) \varepsilon^{n},\left(I-\mathcal{G}_{h}\left(w_{h}^{n}\right)\right)^{2} u^{n}\right) \leq \frac{\chi^{2}}{4}\left\|\varepsilon^{n}\right\|_{\chi, 3}^{2}+\chi^{2}\left\|\left(I-\mathcal{G}_{h}\left(w_{h}^{n}\right)\right) u^{n}\right\|_{\chi, 3}^{2} \\
\chi^{2}\left(\left(I-\mathcal{G}_{h}\left(w_{h}^{n}\right)\right) u^{n},\left(I-\mathcal{G}_{h}\left(w_{h}^{n}\right)\right)^{2} \varepsilon^{n}\right) \leq \chi^{2}\left\|u^{n}\right\|_{\chi, 3}^{2}+\frac{\chi^{2}}{4}\left\|\left(I-\mathcal{G}_{h}\left(w_{h}^{n}\right)\right) \varepsilon^{n}\right\|_{\chi, 3}^{2} \\
2 \chi\left(\left(I-\mathcal{G}_{h}\left(w_{h}^{n}\right)\right) u^{n},\left(I-\mathcal{G}_{h}\left(w_{h}^{n}\right)\right) \varepsilon^{n}\right) \leq \frac{1}{2} \chi\left\|\varepsilon^{n}\right\|_{\chi, 3}^{2}+2 \chi\left\|u^{n}\right\|_{\chi, 3}^{2}
\end{gathered}
$$

Thus, using (4.40)-(4.43) in (4.39), we obtain

$$
\begin{aligned}
& \rho_{\chi, 1}^{2}\left(\varepsilon^{n}\right) \geq \rho_{\chi, 1}^{2}\left(e^{n}\right)+\frac{3}{2} \chi(1-\chi)\left\|\varepsilon^{n}\right\|_{\chi, 3}^{2}+\chi^{2}\left(\left(I-\mathcal{G}_{h}\left(w_{h}^{n}\right)\right) \varepsilon^{n}, \mathcal{G}_{h}\left(w_{h}^{n}\right)\left(I-\mathcal{G}_{h}\left(w_{h}^{n}\right)\right) \varepsilon^{n}\right) \\
& -2 \chi(1+\chi)\left\|u^{n}\right\|_{\chi, 3}^{2}-\chi^{2} \rho_{\chi, 1}^{2}\left(\left(I-\mathcal{G}_{h}\left(w_{h}^{n}\right)\right) u^{n}\right) .
\end{aligned}
$$

\section{NumericAl EXPERIMENTS}

In this section we present four numerical experiments. Using the Green-Taylor vortex problem and selecting regularization by deconvolution $G_{h}(\phi)=D_{h}^{N}(\bar{\phi})$, we confirm the predicted convergence rates and compare the accuracy for deconvolution orders $N=0,1,2$. We then consider the flow around a cylinder, Poisseuille and shear layer benchmark problems, and rotating flow between offset cylinders. Regularizations are required for under resolved flows. Thus, in these tests we have selected flows for which we can obtain a fully resolved, unregularized "truth" solution to compare the coarser mesh, under resolved regularized approximation.

Using FreeFEM++ [22], Algorithm 1.1 is discretized in space using Taylor-Hood elements (continuous piecewise quadratic polynomials for the velocity and continuous linears for the pressure). The nonlinear system at each time step was solved by a fixed point iteration. The Stokes filter and van Cittert deconvolution of orders $N=0,1$ or 2 were used in all the computations. It was applied with the same boundary conditions as given for the problem being solved.

\subsection{Convergence rate verification}

Our first test is designed to test (and does confirm) the predicted rates of convergence. The problem of simulating decay of the Green-Taylor vortex, $[44,45]$, is an interesting test problem in which the true solution is known (which is required to compute the errors to obtain rates of convergence). It is a commonly used 
TABle 1. Poor convergence without relaxation or deconvolution.

\begin{tabular}{ccccc}
\hline$m$ & $\left\|u-u_{h} \mid\right\|_{\infty, 0}$ & Rate & $\left\|\nabla u-\nabla u_{h} \mid\right\|_{2,0}$ & Rate \\
\hline 16 & $2.74021 \times 10^{-2}$ & & $1.64089 \times 10^{-1}$ & \\
32 & $2.61947 \times 10^{-2}$ & 0.06 & $1.50942 \times 10^{-1}$ & 0.12 \\
48 & $2.42064 \times 10^{-2}$ & 0.19 & $1.3133 \times 10^{-1}$ & 0.34 \\
64 & $2.12955 \times 10^{-2}$ & 0.45 & $1.09657 \times 10^{-1}$ & 0.63 \\
80 & $1.78571 \times 10^{-2}$ & 0.79 & $9.03406 \times 10^{-2}$ & 0.87 \\
96 & $1.47115 \times 10^{-2}$ & 1.06 & $7.46497 \times 10^{-2}$ & 1.05 \\
\hline
\end{tabular}

TABLE 2. Errors and convergence rates for deconvolution $N=1, \chi=0$.

\begin{tabular}{ccccc}
\hline$m$ & $\left\|\left|u-u_{h}\right|\right\|_{\infty, 0}$ & Rate & $\left\|\nabla u-\nabla u_{h} \mid\right\|_{2,0}$ & Rate \\
\hline 16 & $2.60813 \times 10^{-2}$ & & $1.52619 \times 10^{-1}$ & \\
32 & $1.4652 \times 10^{-2}$ & 0.83 & $8.08192 \times 10^{-2}$ & 0.96 \\
48 & $5.61527 \times 10^{-3}$ & 2.37 & $4.03054 \times 10^{-2}$ & 1.72 \\
64 & $2.49971 \times 10^{-3}$ & 2.81 & $2.29939 \times 10^{-2}$ & 1.95 \\
80 & $1.27253 \times 10^{-3}$ & 3.03 & $1.44953 \times 10^{-2}$ & 2.07 \\
96 & $7.14506 \times 10^{-4}$ & 3.17 & $9.88172 \times 10^{-3}$ & 2.10 \\
\hline
\end{tabular}

test for accuracy experiments, e.g., $[10,25,43]$. For an insightful analysis see $[5,6]$. The prescribed solution in $\Omega=(0,1) \times(0,1)$ is given by

$$
\begin{aligned}
& u_{1}(x, y, t)=-\cos (\omega \pi x) \sin (\omega \pi y) e^{-2 \omega^{2} \pi^{2} t / \tau}, \\
& u_{2}(x, y, t)=\sin (\omega \pi x) \cos (\omega \pi y) e^{-2 \omega^{2} \pi^{2} t / \tau}, \\
& p(x, y, t)=-\frac{1}{4}(\cos (2 \omega \pi x)+\cos (2 n \pi y)) e^{-2 \omega^{2} \pi^{2} t / \tau} .
\end{aligned}
$$

When $\tau=R e$, this is a solution of the NSE with $f=0$, consisting of an $\omega \times \omega$ array of oppositely signed vortices that decay as $t \rightarrow \infty$.

In our tests we choose $\omega=1, T=1$, Reynolds number $R e=100($ i.e. $\nu=0.01$ ) and $\delta=h=1 / m$, the interval $(0,1)$. The results for Algorithm 1.1 are presented in Table 2, using order of deconvolution $N=1$ without relaxation (i.e. $\chi=0$ ). Tables 3 and 4 present the results for $N=1$ and $N=2$ with relaxation for $\chi=\Delta t$, respectively. Results using the simple averaging filter, i.e. deconvolution with order $N=0$ and $\chi=0$, are presented in Table 1. The convergence rate is calculated from the error at two successive values of $h$ in the usual manner by postulating $e(h)=C h^{\beta}$ and solving for $\beta$ via $\beta=\ln \left(e\left(h_{1}\right) / e\left(h_{2}\right)\right) / \ln \left(h_{1} / h_{2}\right)$.

From the tables we see the convergence rate approaches the second order rate predicted for $\left\|\left|\nabla u-\nabla u_{h}\right|\right\|_{2,0}$ and we also see what appears to be an $L^{2}$ lift for $\left\|\left|u-u_{h}\right|\right\|_{\infty, 0}$ for order of deconvolution $N=1$ and $N=2$. The method with the simple averaging filter, order of deconvolution $N=0$, has much larger errors and slower rates of convergence, as expected. From this test it is clear that (i) relaxation is important to control the loss of accuracy due to blow up of the numerical dissipation as $\Delta t \rightarrow 0$, and (ii) regularization using filtering plus deconvolution is superior to low order filtering alone, as predicted in Remark 4.3.

\subsection{Flow around a cylinder}

Our next numerical illustration is for two dimensional under-resolved flow around a cylinder. Thus, our goal is not to use a fine mesh and reproduce the benchmark values from $[24,36]$ but rather to see how close to those values we can come on a mesh coarse enough that accuracy cannot be reasonably expected. We compute values for the maximal $\operatorname{drag} c_{d, \max }$ and lift $c_{l, \max }$ coefficient at the cylinder, and for the pressure difference 
TABLE 3. Errors and convergence rates for deconvolution $N=1$ with $\chi=\Delta t$.

\begin{tabular}{ccccc}
\hline$m$ & $\left\|u-u_{h} \mid\right\|_{\infty, 0}$ & Rate & $\left\|\nabla u-\nabla u_{h} \mid\right\|_{2,0}$ & Rate \\
\hline 16 & $1.00069 \times 10^{-3}$ & & $1.61231 \times 10^{-2}$ & \\
32 & $1.48731 \times 10^{-4}$ & 2.75 & $2.62388 \times 10^{-3}$ & 2.62 \\
48 & $4.19167 \times 10^{-5}$ & 3.12 & $9.44449 \times 10^{-4}$ & 2.52 \\
64 & $1.63416 \times 10^{-5}$ & 3.27 & $4.71362 \times 10^{-4}$ & 2.42 \\
80 & $7.71333 \times 10^{-6}$ & 3.36 & $2.8071 \times 10^{-4}$ & 2.32 \\
96 & $4.12874 \times 10^{-6}$ & 3.43 & $1.86365 \times 10^{-4}$ & 2.25 \\
\hline
\end{tabular}

TABLE 4. Errors and convergence rates for deconvolution $N=2$ with $\chi=\Delta t$.

\begin{tabular}{ccccc}
\hline$m$ & $\left\|u-u_{h} \mid\right\|_{\infty, 0}$ & Rate & $\left\|\nabla u-\nabla u_{h} \mid\right\|_{2,0}$ & Rate \\
\hline 16 & $4.98747 \times 10^{-4}$ & & $1.54581 \times 10^{-2}$ & \\
32 & $4.63728 \times 10^{-5}$ & 3.43 & $2.38295 \times 10^{-3}$ & 2.70 \\
48 & $1.0654 \times 10^{-5}$ & 3.63 & $8.57864 \times 10^{-4}$ & 2.52 \\
64 & $3.66425 \times 10^{-6}$ & 3.71 & $4.34974 \times 10^{-4}$ & 2.36 \\
80 & $1.59044 \times 10^{-6}$ & 3.74 & $2.6326 \times 10^{-4}$ & 2.25 \\
96 & $8.04969 \times 10^{-7}$ & 3.73 & $1.77074 \times 10^{-4}$ & 2.18 \\
\hline
\end{tabular}

$\Delta p(t)$ between the front and back of the cylinder at the final time $T=8$. It is not turbulent but does have interesting features. The flow patterns are driven by the interaction of a fluid with a wall which is an important scenario for industrial flows. This flow is actually quite difficult to simulate successfully by a model with sufficient regularization to handle higher Reynolds number problems.

The time dependent inflow profile is

$$
\begin{aligned}
& u_{1}(0, y, t)=u_{1}(2.2, y, t)=\frac{6}{0.41^{2}} \sin (\pi t / 8) y(0.41-y) \\
& u_{2}(0, y, t)=u_{2}(2.2, y, t)=0
\end{aligned}
$$

No slip boundary conditions are prescribed along the top and bottom walls, "do-nothing" at the outflow, and the initial condition is $u(x, y, 0)=0$. The viscosity is $\nu=10^{-3}$ and the external force $f=0$. The Reynolds number of the flow, based on the diameter of the cylinder and on the mean velocity inflow is $0 \leq R e \leq 100$. The domain is a rectangle $2.2 \times 0.41$, with circle centered at $(0.2,0.2)$ of radius 0.05 . A mesh with 62757 total number of degrees of freedom (velocity and pressure) is used for all simulation for a clear comparison of the different parameter settings presented in this report. The filter radius is chosen as the perimeter of the cylinder divided by the number of mesh points around the cylinder.

From time $t=2$ to $t=4$ two vortices start to develop behind the cylinder. Between $t=4$ and $t=5$, the vortices separate from the cylinder, so that a vortex street develops, and they continue to be visible through the final time $t=8$. This can be seen in Figure 1. The evolutions of $c_{d, \max }, c_{l, \max }$ and $\Delta p$ are presented in Figure 2.

For the computation of drag and lift coefficients fd the one dimensional method described by John [24]. Results on the computations of maximal drag and lift coefficients and pressure drop, for $N=1$, are presented in Table 5. The following reference intervals are given in [36]

$$
c_{d, \max }^{\mathrm{ref}} \in[2.93,2.97], \quad c_{l, \max }^{\mathrm{ref}} \in[0.47,0.49], \quad \Delta p^{\mathrm{ref}} \in[-0.115,-0.105]
$$

and also the following reference values are given in [24] 

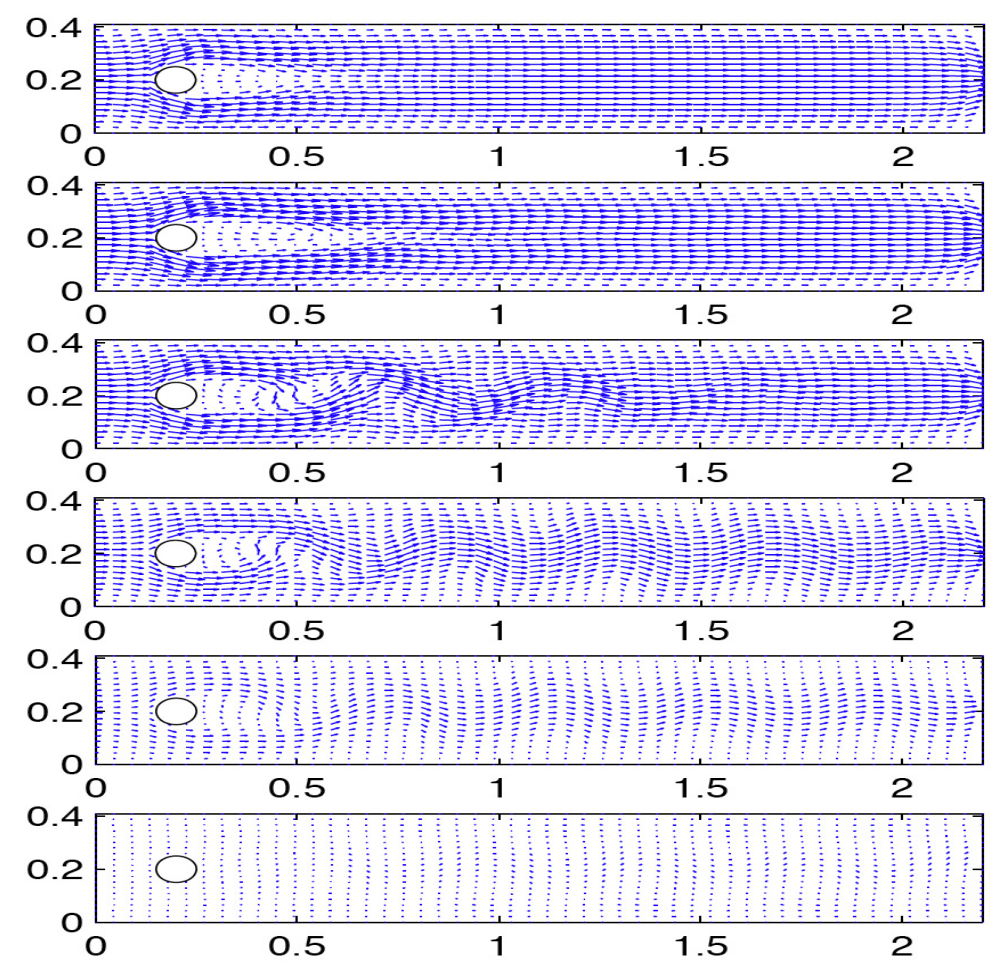

Figure 1 . The velocity at $\mathrm{t}=2,4,5,6,7$, and 8 of Algorithm 1.1 , with $N=1$ and $\chi=\Delta t=$ 0.005 .

TABLE 5. Drag/lift coefficients and pressure difference for $N=1$ deconvolution.

\begin{tabular}{lcccccc}
\hline Relax. coeff. & $\Delta t$ & $\mathrm{t}\left(c_{d, \max }\right)$ & $c_{d, \max }$ & $\mathrm{t}\left(c_{l, \max }\right)$ & $c_{l, \max }$ & $\Delta p(8 \mathrm{~s})$ \\
\hline \multirow{4}{*}{$\chi=0$} & 0.02 & 3.94 & 2.81978 & 6.14 & 0.320677 & -0.109436 \\
& 0.01 & 3.93 & 2.75983 & 6.02 & 0.366276 & -0.0996473 \\
& 0.005 & 3.925 & 2.66524 & 6.03 & 0.325459 & -0.098704 \\
\hline \multirow{3}{*}{$\chi=\Delta t$} & 0.02 & 3.94 & 2.94149 & 6.12 & 0.360383 & -0.106385 \\
& 0.01 & 3.93 & 2.94231 & 5.96 & 0.454216 & -0.108634 \\
& 0.005 & 3.935 & 2.94268 & 5.925 & 0.477011 & -0.111908 \\
\hline
\end{tabular}

$$
\begin{aligned}
& t\left(c_{d, \text { max }}^{\mathrm{ref}}\right)=3.93625, \quad c_{d, \text { max }}^{\mathrm{ref}}=2.950921575 \\
& t\left(c_{l, \text { max }}^{\mathrm{ref}}\right)=5.693125, \quad c_{l, \text { max }}^{\mathrm{ref}}=0.47795 \\
& \Delta p^{\mathrm{ref}}(8 s)=-0.1116
\end{aligned}
$$

Table 5 shows that using a regularization operator in Step 1.1 of Algorithm 1.1 works well in combination with the BDF2 time discretization in Algorithm 1.1. It computes the drag and lift coefficients, and the pressure difference, within the benchmark intervals, and illustrates the positive role of using relaxation in the approximation algorithm. 

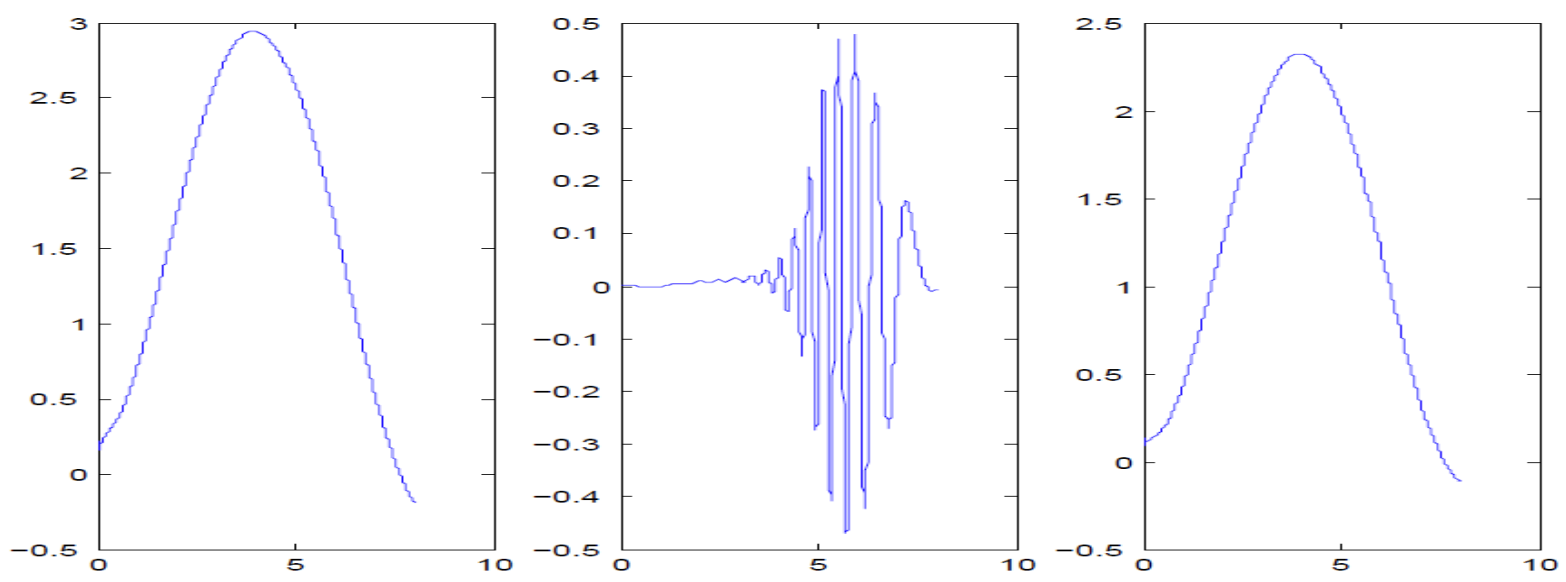

Figure 2. The development of $c_{d}(t), c_{l}(t)$ and $\Delta p(t)$ of Algorithm 1.1 with $N=1$ and $\chi=$ $\Delta t=0.005$.

\subsection{Poisseuille flow}

A discussion of this problem can be found in Canuto, Hussaini, Quarteroni, and Zang [9]. The goal of this test is to test the contribution of the BDF2 discretization in Step 1.1 by comparing the sensitivity to perturbations over longer time intervals and higher Re of Algorithm 1.1 to Algorithm 1.1 with Step 1.1 replaced by the CN method, studied in [17]. To do so we initialize with a linearly stable equilibrium solution and take many steps with a large $\Delta t$ to check for deviations from equilibrium. At each time step there are small perturbations due to discretization effects. Thus we test if this linearly stable flow remains linearly stable under CN versus BDF2 methods used in Step 1.1 of Algorithm 1.1.

The results show that BDF2 increases the stability of Algorithm 1.1 over the CN time discretization. In $\Omega=(-1,1) \times(-0.5,0.5)$, a parabolic velocity $v(x, t)=0$ and $u(x, y, t)=(y+0.5)(0.5-y)$ is prescribed at the inflow and outflow. No-slip boundary conditions are given at the top and bottom. The exact solution is well known to be $v(x, y)=0, u(x, y)=(y+0.5)(0.5-y), p(x, y)=-2 \nu x$, and we take it as our initial condition. We take the viscosity $\nu=10^{-5}$, filter width $\delta=0.1$, order of deconvolution $N=1$ and relaxation parameter $\chi=\Delta t$. A uniform mesh consisting of triangles with 1953 number of degrees of freedom was used.

For time step $\triangle t=1$, the results of the velocity fields after 123 time steps show that Algorithm 1.1 is both more accurate and less sensitive to perturbation. At $T=123$ using CN in Algorithm 1.1 the flow lost its features, see Figure 3, while the velocity field computed by the Algorithm 1.1 is properly simulated even at $T=200$, see Figure 4.

Relative velocity errors for the given initial condition with time step $\Delta t=0.5$ and 1 and relative velocity errors for a perturbed initial condition $v(x, y)=0, u(x, y)=(y+0.5)(0.5-y)+0.001 \sin (4 \pi y)$ with time step $\triangle t=0.5$ and 1 are given in Figures 5 and 6 from left to right, respectively.

The velocity errors from the CN method in Step 1.1 are larger, while the ones with BDF2 method in 1.1 are smaller, especially in the left plot of Figure 5, where the error curve for BDF2 is very close to the horizontal-axis, and thus hard to observe on the graph.

\subsection{Shear layer roll-up problem}

This is a benchmark problem studied in [18]. The domain is $\Omega=[0,1]^{2}$ the unit square, initial conditions are

$$
u=\tanh (\rho(y-0.25)) \text { for } y \leq 0.5, \quad u=\tanh (\rho(0.75-y)) \text { for } y>0.5, \quad v=0.05 \sin (2 \pi x),
$$




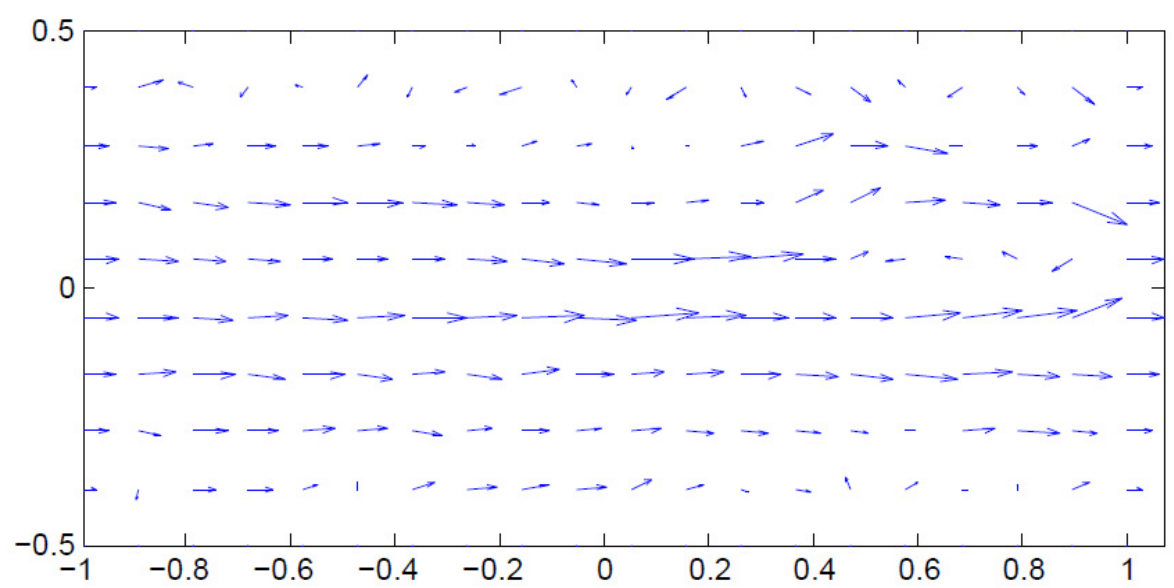

FiguRE 3. Incorrect velocity field when Step 1.1 uses Crank-Nicolson.

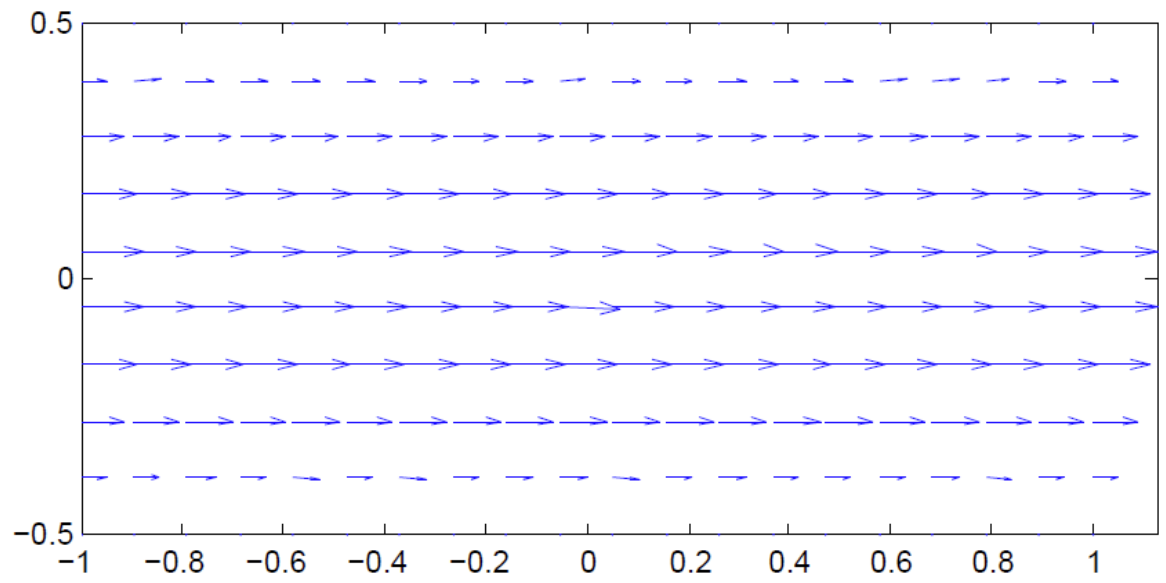

Figure 4. Correct velocity field when Step 1.1 uses BDF2.

and doubly-periodic boundary conditions are applied on $\Omega$. The time step $\triangle t=0.002$, Reynolds number $R e=40000$ (i.e. $\nu=1 / 40000$ ), Taylor-Hood finite elements, and uniform triangular mesh, were used in all cases. The relaxation parameter $\chi=\Delta t$, while the filter radius $\delta=1 / m$, where $m$ denotes the number of subintervals on each side of the square domain $\Omega$. We present the true unfiltered vorticity solution (i.e. Navier-Stokes solution) obtained on a fine mesh with $m=170$, then, the rest of the plots in Figure 7 are on a coarse mesh with $m=90$. All the presented plots are at the final time $T=1.2$. These vorticity plots show the advantage of applying filtering/deconvolution and relaxation step (i.e. steps $2 \mathrm{a}$ and $2 \mathrm{~b}$ of the Algorithm 1.1) in comparison with no filtering, i.e. Navier-Stokes solution that has lots of spurious vortices on the coarse mesh.

\subsection{Rotating flow between offset cylinders}

Finally we compare four options (CN without Step 1.1, BDF2 without Step 1.1, BDF2 with N=1 deconvolutions in Step 1.1 and BDF2 with $N=2$ deconvolutions in Step 1.1) on a problem motivated by the classic 

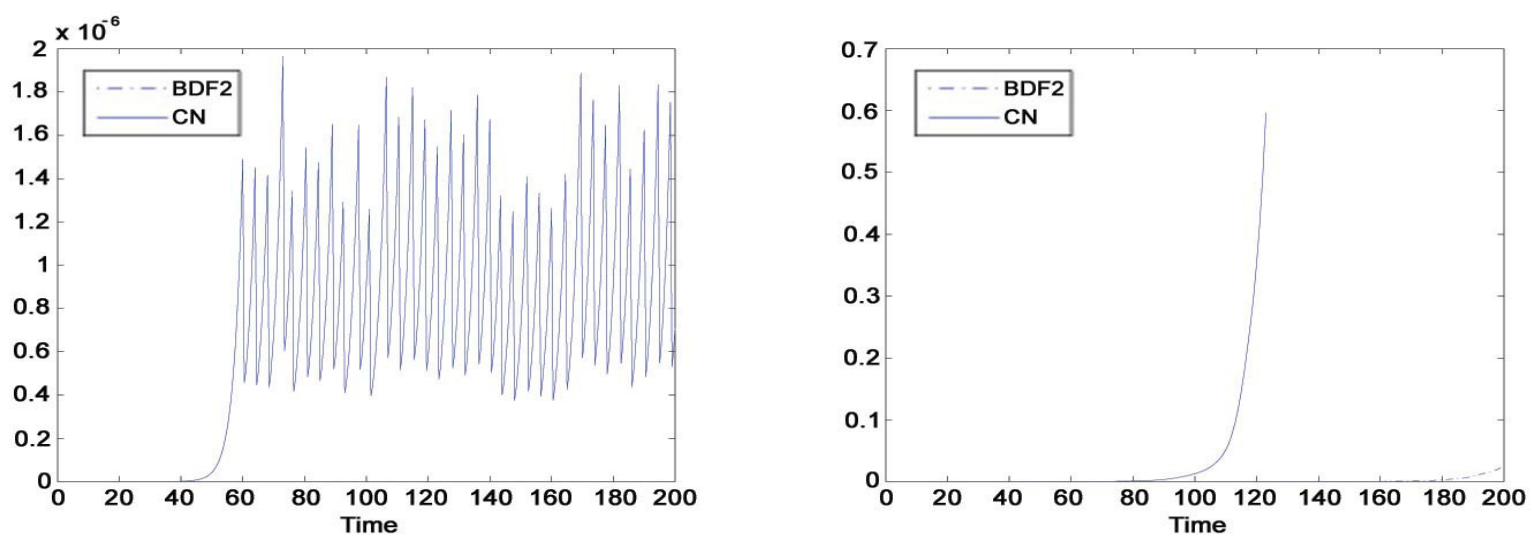

FiguRE 5. Relative velocity error vs. time $\Delta t=0.5, \Delta t=1$. From left to right, CN: large, and BDF2: error close to $x$-axis (very small).
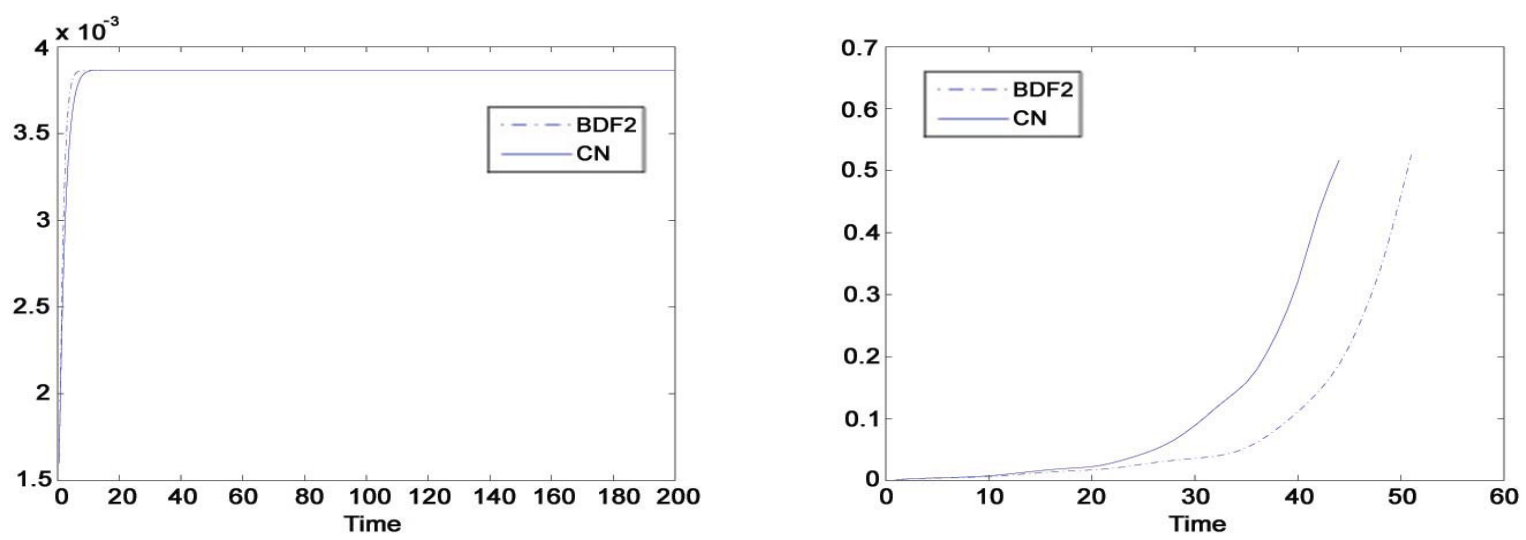

FIgURE 6. Relative velocity error vs. time for $\Delta t=0.5, \Delta t=1$ with perturbed initial condition.

problem of flow between rotating cylinders. We take the domain to be a disk with a smaller, off center, obstacle inside. Let $r_{1}=1, r_{2}=0.1, c=\left(c_{1}, c_{2}\right)=\left(\frac{1}{2}, 0\right)$,

$$
\Omega=\left\{(x, y): x^{2}+y^{2} \leq r_{1}^{2} \text { and }\left(x-c_{1}\right)^{2}+\left(y-c_{2}\right)^{2} \geq r_{2}^{2}\right\} .
$$

The boundary conditions are no slip, $R e=250$ and the flow is driven by a body force (rather than rotation of either cylinder)

$$
f(x, y)=(-2 y, 2 x)
$$

which induces a counter clockwise rotation. The flow rotates about $(0,0)$, interacts with the immersed cylinder $\left(x-c_{1}\right)^{2}+\left(y-c_{2}\right)^{2} \leq r_{2}^{2}$ which induces a von Kármán vortex street. This vortex street rotates and itself re-interacts with the immersed cylinder, creating more complex structures. This flow also contains complex structures in the boundary layer near $x^{2}+y^{2}=r_{1}^{2}$ which are not resolved on the mesh. (To resolve these would require a mesh with at least 3 points within $O(1 / R e)=O(1 / 250)$ of each wall.) The mesh is parameterized by the number of mesh points around the outer cylinder $(n=40$ and 60$)$ and $\frac{n}{4}$ mesh points around the immersed cylinder, and extended to all of $\Omega$ as a Delaunay mesh. ${ }^{5}$

\footnotetext{
${ }^{5}$ An expanded version of this report is available at http://www.mathematics.pitt.edu/research/technical-reports. phphttp://www.mathematics.pitt.edu/research/technical-reports.php containing plots of the $n=20$ case and plots of energy vs. time for $n=40$ and 60 .
} 


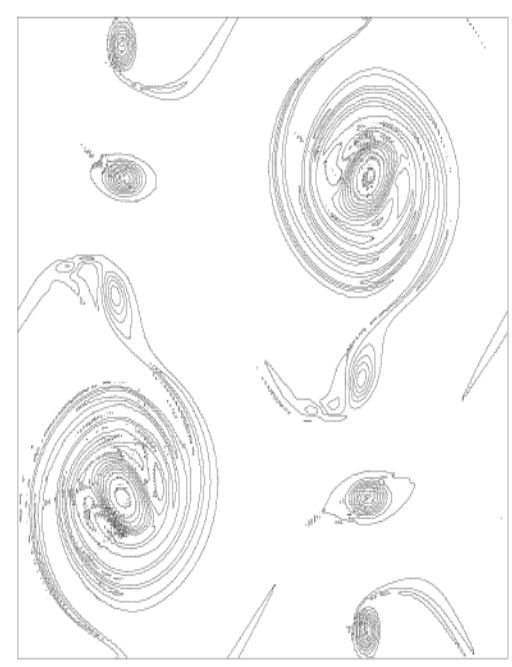

(a) NSE, fine mesh

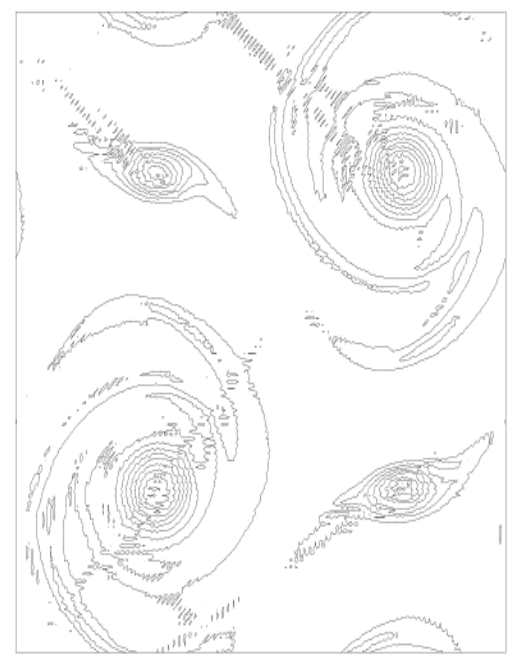

(c) deconvolution $N=0, \chi=\triangle t$ coarse mesh

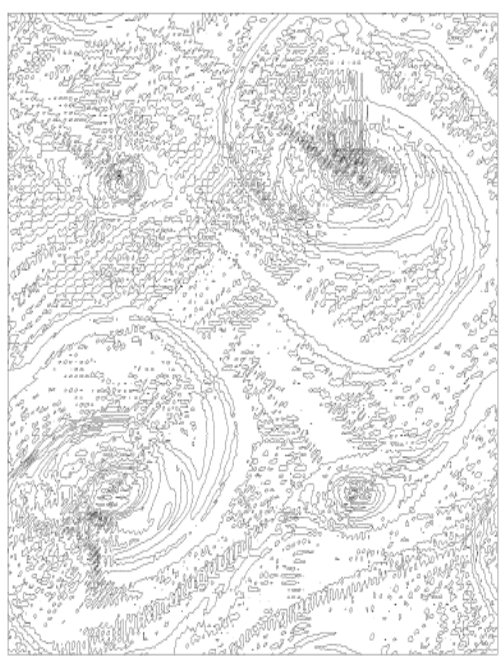

(b) NSE, coarse mesh

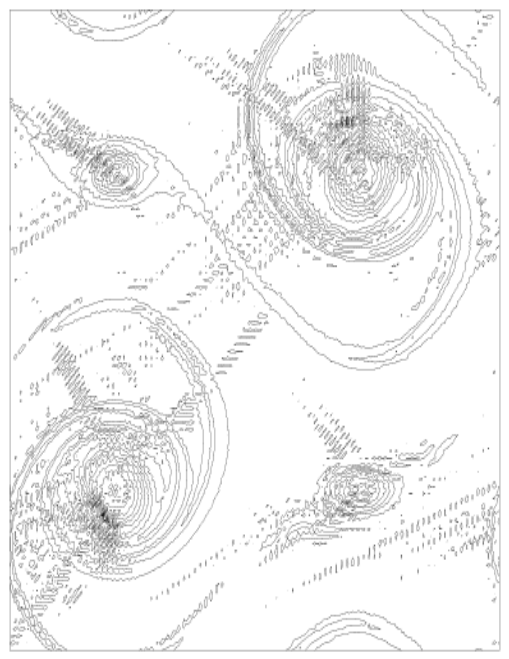

(d) deconvolution $N=1, \chi=\triangle t$, coarse

FiguRE 7. Vorticity for different settings, at $R e=40000$, and $T=1.2$.

We give plots over $0 \leq t \leq 40$ of the following quantities:

$$
\begin{aligned}
& A(t):=\|\boldsymbol{x} \cdot \boldsymbol{u}\| \doteq \text { angular momentum, } \\
& \|\operatorname{curl} \boldsymbol{u}\|=\text { RMS vorticity, } \\
& \|\operatorname{grad} \boldsymbol{u}\|^{2}=\frac{1}{\nu} * \text { enstrophy. }
\end{aligned}
$$

All three are inviscid invariants of $2 d$ flows without boundaries and are interesting for rotational flows. We selected $\chi=\Delta t=0.01$. Our observations on this interesting flow are preliminary, not intended to describe flow details and only intended to test the effects of Step 1.1 on the global balance in three important quantities. 

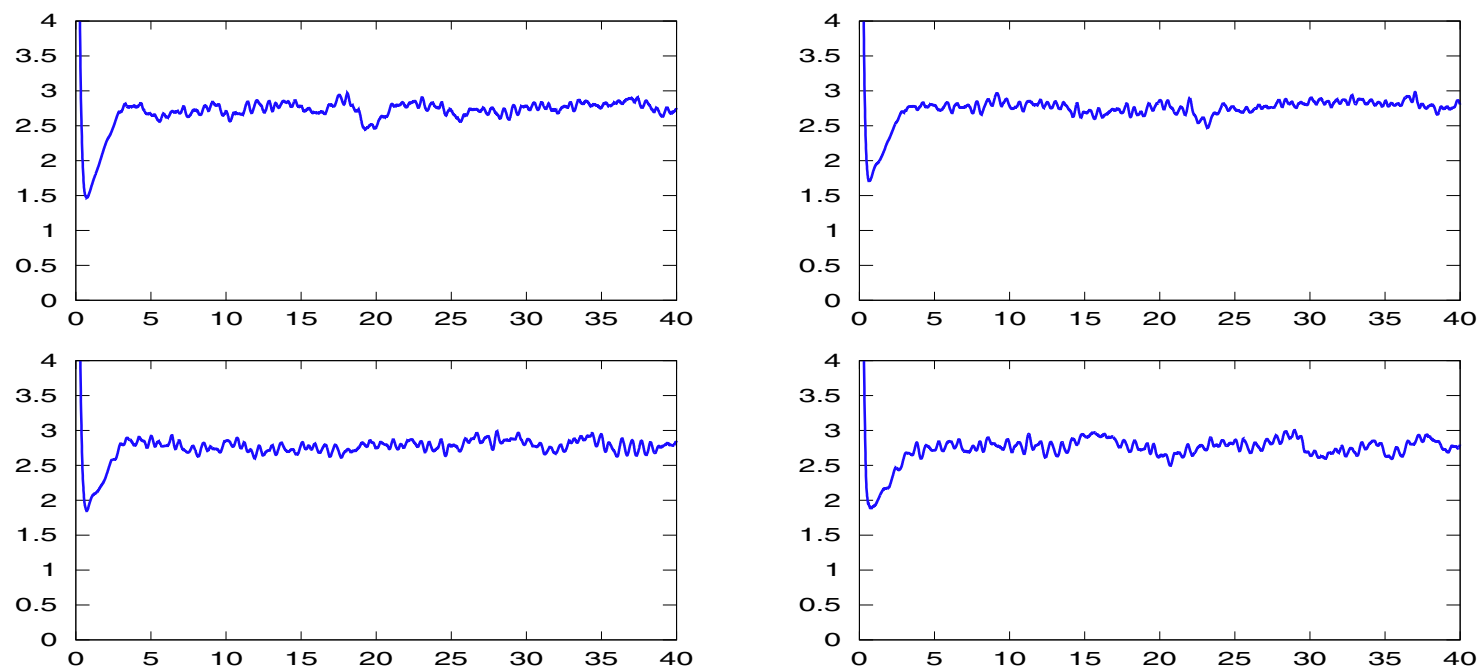

Figure 8. Angular momentum for CN (top left), BDF2 (top right), BDF2 with $N=1$ (bottom left), and BDF2 with $N=2$ (bottom right) calculated with Re $=250, n=40$ mesh points around outer cylinder.
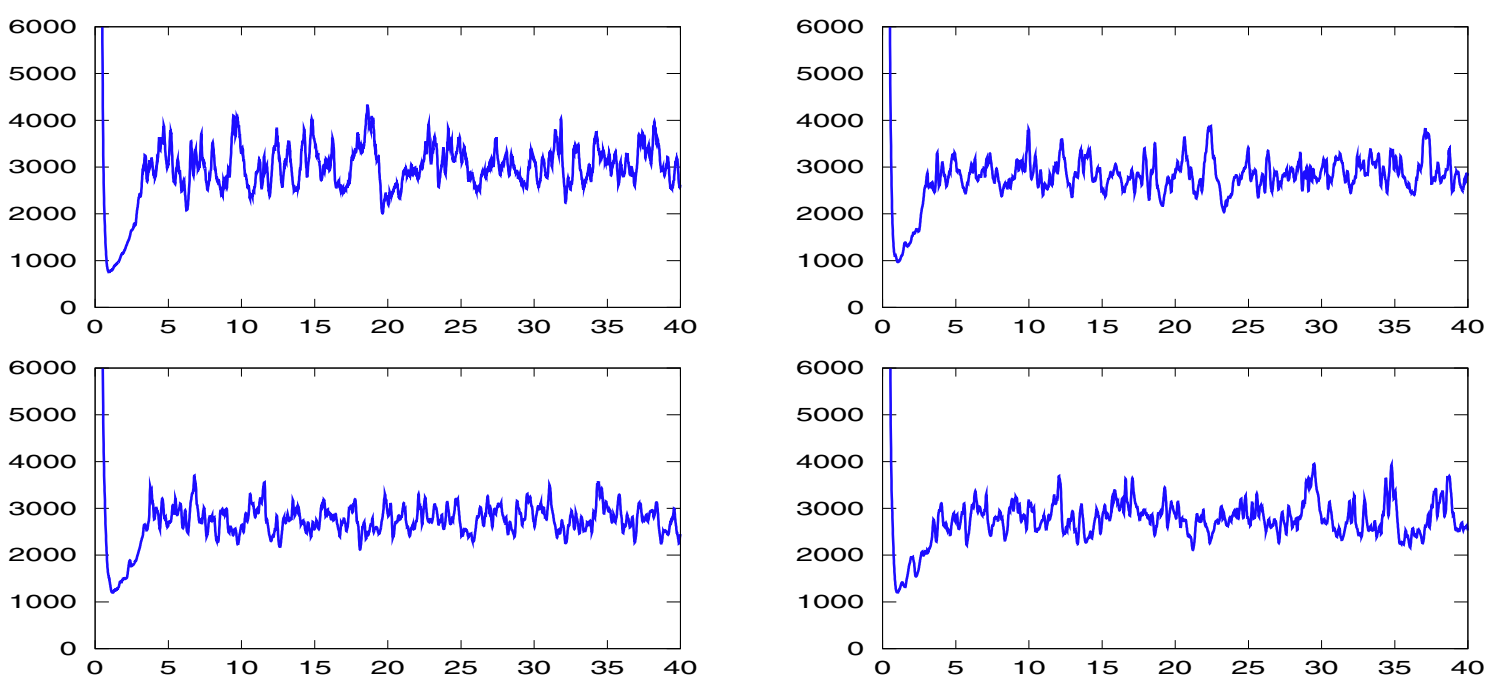

Figure 9. $\|\operatorname{grad} \boldsymbol{u}\|^{2}$, for CN (top left), BDF2 (top right), BDF2 with $N=1$ (bottom left), and BDF2 with $N=2$ (bottom right) calculated with $R e=250, n=40$ mesh points around outer cylinder.

Our goal here is to test if Step 1.1 and the BDF2 time discretization over diffuse rotational quantities in this flow. We begin with the unregularized, centered, conservative Crank-Nicolson (CN) time discretization and meshes with $n=20,40,60$ points around the outer cylinder. For the step $n=20$ (not shown) to $n=40$ $(A(t)$ in Fig. 8, enstrophy in Fig. 9), the angular momentum, enstrophy and total (RMS) vorticity increased by $40 \%$ to $50 \%$. For $n=40$ to $n=60$, the increase in each was less than $5 \%$. We take this as evidence that the $n=40$ (and $n=60$ ) mesh is adequate to represent the global, net amount of each of these rotational quantities. Following this, we repeated the $n=40$ and $n=60$ tests with BDF2 with Step 1.1 regularization 

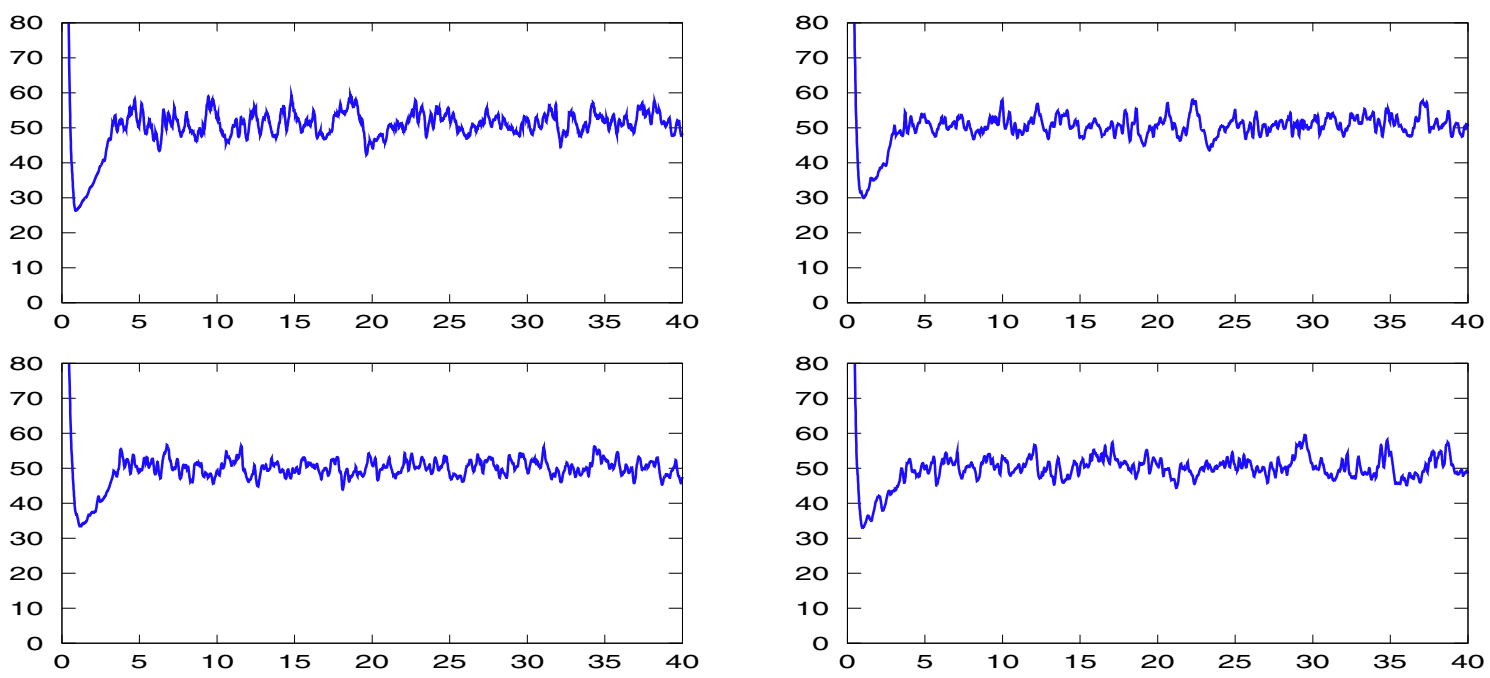

FiguRe 10. Vorticity for CN (top left), BDF2 (top right), BDF2 with $N=1$ (bottom left), and BDF2 with $N=2$ (bottom right) calculated with $R e=250, n=40$ mesh points around outer cylinder.
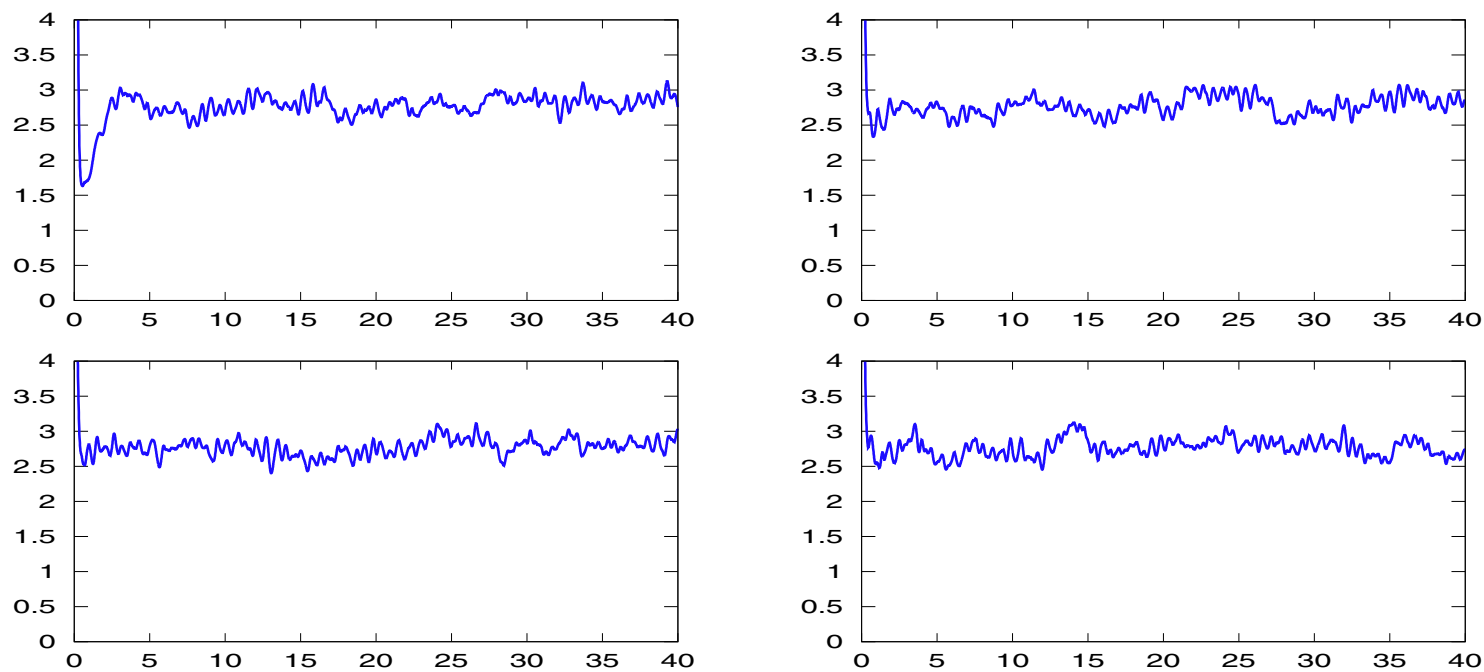

Figure 11. Angular momentum, for CN (top left), BDF2 (top right), BDF2 with $N=1$ (bottom left), and BDF2 with $N=2$ (bottom right) calculated with $R e=250, n=60$ mesh points around outer cylinder.

and without regularization. The results (RMS vorticity in Figs. 10, 13, and enstrophy in Figs. 9 and 12) of the global, net amounts of these same quantities are consistent to the $n=40$ and $n=60 \mathrm{CN}$ tests. (The details are not identical. For example, for BDF2 plus regularization, enstrophy increased by $2 \%$ from the $n=40$ to $n=60$ meshes.) This is evidence that neither BDF2 nor the regularization in Step 1.1 over diffused rotational quantities. Further, without regularization, both CN and BDF2 show initial transients in all quantities (see Figs. 8 through 13) which are nearly eliminated when Step 1.1 is included. 

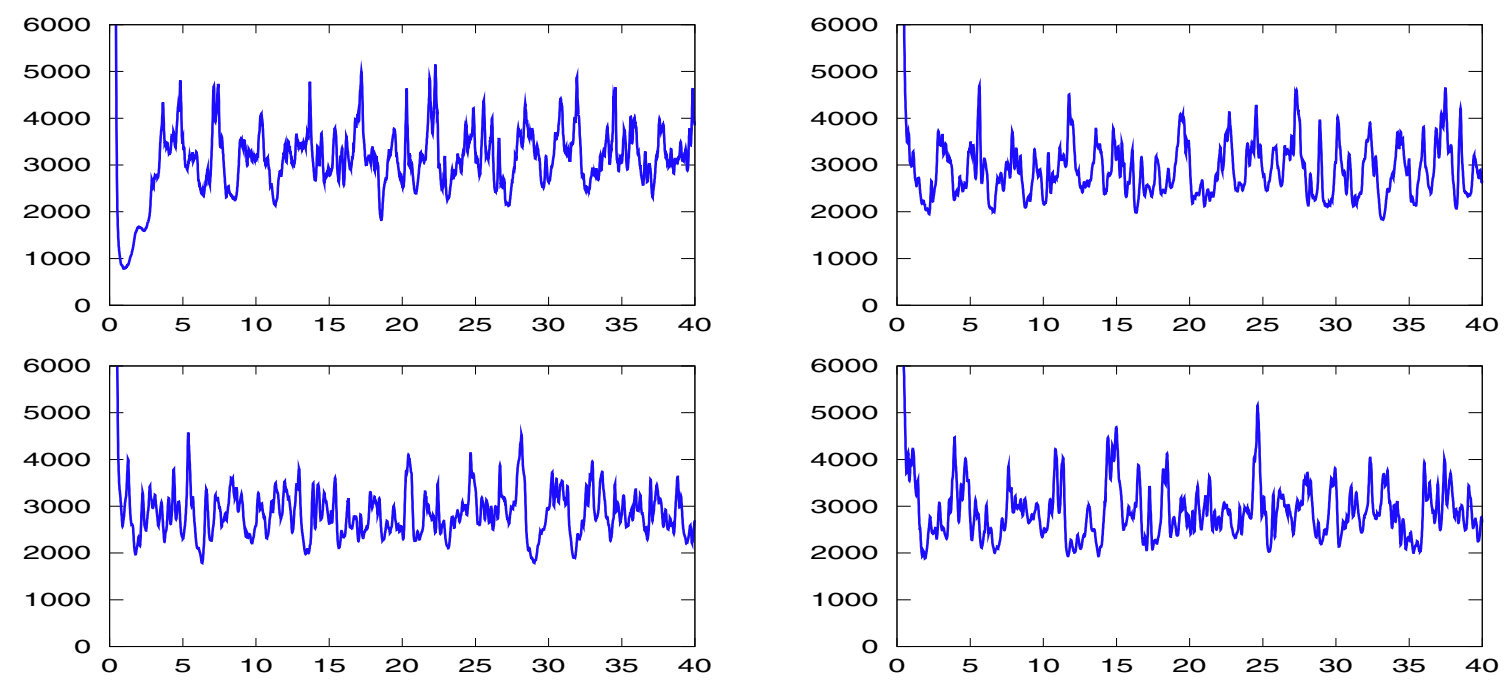

Figure 12. $\|\operatorname{grad} \boldsymbol{u}\|^{2}$ for CN (top left), BDF2 (top right), BDF2 with $N=1$ (bottom left), and BDF2 with $N=2$ (bottom right) calculated with $R e=250, n=60$ mesh points around outer cylinder.
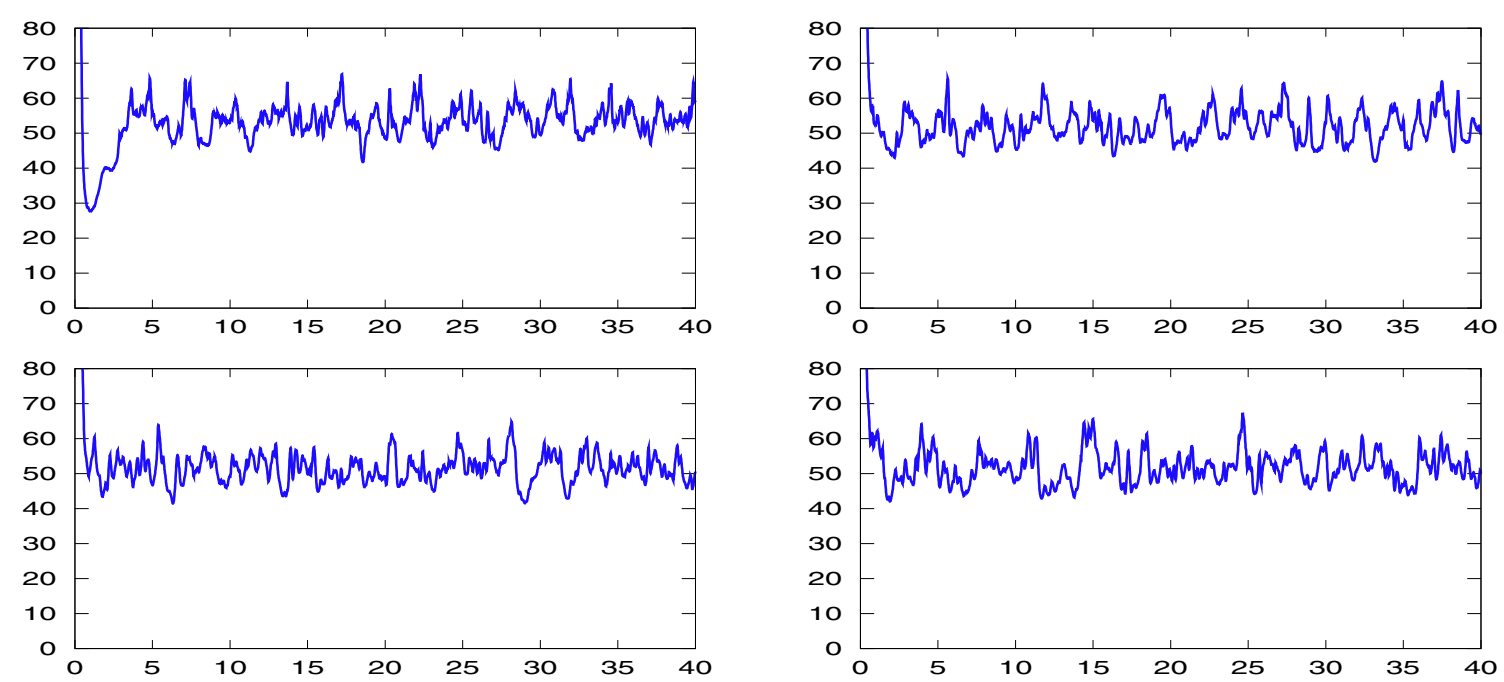

Figure 13. Vorticity for CN (top left), BDF2 (top right), BDF2 with $N=1$ (bottom left), and BDF2 with $N=2$ (bottom right) calculated with $R e=250, n=60$ mesh points around outer cylinder.

\section{Conclusions}

The BDF2 time discretization is second order, A-stable and has stability properties that are superior to those of Crank-Nicolson for underresolved flow simulations. We have seen that modular stabilization by filter, then stabilize works well in combination with BDF2. The numerical experiments confirm the stability and convergence theory and show that this combination is much better than unstabilized methods and somewhat better than Crank-Nicolson plus the same stabilization. 
The correct scaling of the relaxation parameter $\chi$ seems to be $\chi=O(\Delta t)$. However, the precise determination of $\chi$ step by step so as to match numerical dissipation to that occurring on unresolved scales is an important open problem.

Acknowledgements. We thank Dr. Myron Sussman and Dr. F. Hecht for their constructive comments which improved Section 5. This research was partially supported by the NSF under grants DMS-0810385 and DMS-126465 (W. Layton, N. Mays) and by the Air Force under grant FA9550-09-1-0058 (C. Trenchea).

\section{REFERENCES}

[1] N. Adams and A. Leonard, Deconvolution of subgrid scales for the simulation of shock-turbulence interaction, in Direct and Large Eddys Simulation III, edited by N.S.P. Voke and L. Kleiser. Kluwer, Dordrecht (1999) 201.

[2] N. Adams and S. Stolz, Deconvolution methods for subgrid-scale approximation in large-eddy simulation, Modern Simulation Strategies for Turbulent Flow, edited by R.T. Edwards (2001).

[3] N. Adams and S. Stolz, A subgrid-scale deconvolution approach for shock capturing. J. Comput. Phys. 178 (2002) $391-426$.

[4] G.A. Baker, V.A. Dougalis and O.A. Karakashian, On a higher order accurate fully discrete Galerkin approximation to the Navier-Stokes equations. Math. Comput. 39 (1982) 339-375.

[5] D. Barbato, L.C. Berselli and C.R. Grisanti, Analytical and numerical results for the rational large eddy simulation model. J. Math. Fluid Mech. 9 (2007) 44-74.

[6] L.C. Berselli, On the large eddy simulation of the Taylor-Green vortex. J. Math. Fluid Mech. 7 (2005) S164-S191.

[7] J.P. Boyd, Two comments on filtering (artificial viscosity) for Chebyshev and Legendre spectral and spectral element methods: preserving boundary conditions and interpretation of the filter as a diffusion. J. Comput. Phys. 143 (1998) $283-288$.

[8] S.C. Brenner and L.R. Scott, The mathematical theory of finite element methods, vol. 15 of Texts in Applied Mathematics. Springer-Verlag, New York (1994).

[9] C. Canuto, M.Y. Hussaini, A. Quarteroni and T.A. Zang, Spectral methods, Evolution to complex geometries and applications to fluid dynamics. Scientific Computation. Springer, Berlin (2007).

[10] A. Chorin, Numerical solution for the Navier-Stokes equations. Math. Comput. 22 (1968) 745-762.

[11] J. Connors and W. Layton, On the accuracy of the finite element method plus time relaxation. Math. Comput. 79 (2010) 619-648.

[12] A. Dunca, Investigation of a shape optimization algorithm for turbulent flows, tech. rep., Argonne National Lab, report number ANL/MCS-P1101-1003 (2002). Available at http://www-fp.mcs.anl.gov/division/publications/.

[13] A. Dunca, Space averaged Navier Stokes equations in the presence of walls. Ph.D. thesis, University of Pittsburgh (2004).

[14] A. Dunca and Y. Epshteyn, On the Stolz-Adams deconvolution model for the large-eddy simulation of turbulent flows. SIAM J. Math. Anal. 37 (2006) 1890-1902.

[15] E. Emmrich, Error of the two-step BDF for the incompressible Navier-Stokes problem. M2AN: M2AN 38 (2004) $757-764$.

[16] V. Ervin, W. Layton and M. Neda, Numerical analysis of a higher order time relaxation model of fluids. Int. J. Numer. Anal. Model. 4 (2007) 648-670.

[17] V. Ervin, W. Layton and M. Neda, Numerical analysis of filter based stabilization for evolution equations. SINUM 50 (2012) $2307-2335$.

[18] P. Fischer and J. Mullen, Filter-based stabilization of spectral element methods. C. R. Acad. Sci. Paris Sér. I Math. 332 (2001) 265-270.

[19] E. Garnier, N. Adams and P. Sagaut, Large eddy simulation for compressible flows. Sci. Comput. Springer, Berlin (2009).

[20] V. Girault and P.-A. Raviart, Finite element approximation of the Navier-Stokes equations, in vol. 749 of Lect. Notes Math. Springer-Verlag, Berlin (1979).

[21] M.D. Gunzburger, Finite element methods for viscous incompressible flows, A guide to theory, practice, and algorithms. Computer Science and Scientific Computing. Academic Press Inc., Boston, MA (1989).

[22] F. Hecht and O. Pironneau, Freefem++, webpage: http://www.freefem.org.

[23] J.G. Heywood and R. Rannacher, Finite-element approximation of the nonstationary Navier-Stokes problem. IV. Error analysis for second-order time discretization. SIAM J. Numer. Anal. 27 (1990) 353-384.

[24] V. John, Large eddy simulation of turbulent incompressible flows, Analytical and numerical results for a class of LES models, in vol. 34 of Lect. Notes Comput. Sci. Engrg. Springer-Verlag, Berlin (2004).

[25] V. John and W.J. Layton, Analysis of numerical errors in large eddy simulation. SIAM J. Numer. Anal. 40 (2002) 995-1020.

[26] W. Layton, Superconvergence of finite element discretization of time relaxation models of advection. BIT 47 (2007) 565-576.

[27] W. Layton, The interior error of van Cittert deconvolution is optimal. Appl. Math. 12 (2012) 88-93.

[28] W. Layton, C. Manica, M. Neda and L. Rebholz, Helicity and energy conservation and dissipation in approximate deconvolution LES models of turbulence. Adv. Appl. Fluid Mech. 4 (2008) 1-46.

[29] W. Layton and M. Neda, Truncation of scales by time relaxation. J. Math. Anal. Appl. 325 (2007) $788-807$.

[30] W. Layton, L.G. Rebholz and C. Trenchea, Modular nonlinear filter stabilization of methods for higher Reynolds numbers flow. J. Math. Fluid Mech. (2011) 1-30. 
[31] W. Layton, L. Röhe and H. Tran, Explicitly uncoupled VMS stabilization of fluid flow. Comput. Methods Appl. Mech. Engrg. 200 (2011) 3183-3199.

[32] J. Mathew, R. Lechner, H. Foysi, J. Sesterhenn and R. Friedrich, An explicit filtering method for large eddy simulation of compressible flows. Phys. Fluids 15 (2003).

[33] J.S. Mullen and P.F. Fischer, Filtering techniques for complex geometry fluid flows. Commun. Numer. Methods Engrg. 15 (1999) 9-18.

[34] S. Ravindran, Convergence of extrapolated BDF2 finite element schemes for unsteady penetrative convection model. Numer. Funct. Anal. Optim. 33 (2011) 48-79.

[35] P. Rosenau, Extending hydrodynamics via the regularization of the Chapman-Enskog expansion. Phys. Rev. A 40 (1989) 7193.

[36] M. Schäfer and S. Turek, Benchmark computations of laminar flow around cylinder, in Flow Simulation with High-Performance Computers II, vol. 52. Edited by H. EH. Vieweg (1996) 547-566.

[37] S. Schochet and E. Tadmor, The regularized Chapman-Enskog expansion for scalar conservation laws. Arch. Rat. Mech. Anal. 119 (1992) 95.

[38] I. Stanculescu, Existence theory of abstract approximate deconvolution models of turbulence. Ann. Univ. Ferrara Sez. VII Sci. Mat. 54 (2008) 145-168.

[39] S. Stolz and N. Adams, On the approximate deconvolution procedure for LES. Phys. Fluids, II (1999) $1699-1701$.

[40] S. Stolz, N. Adams and L. Kleiser, An approximate deconvolution model for large eddy simulation with application to wallbounded flows. Phys. Fluids 13 (2001) 997-1015.

[41] S. Stolz, N. Adams and L. Kleiser, The approximate deconvolution model for LES of compressible flows and its application to shock-turbulent-boundary-layer interaction. Phys. Fluids 13 (2001) 2985.

[42] S. Stolz, N. Adams and L. Kleiser, The approximate deconvolution model for compressible flows: isotropic turbulence and shock-boundary-layer interaction, Advances in LES of Complex Flows, in vol. 65 of Fluid Mechanics and Its Applications. Edited by R. Friedrich and W. Rodi. Springer, Netherlands (2002) 33-47.

[43] D. Tafti, Comparison of some upwind-biased high-order formulations with a second-order central-difference scheme for time integration of the incompressible Navier-Stokes equations. Comput. Fluids 25 (1996) 647-665.

[44] G. Taylor, On decay of vortices in a viscous fluid. Phil. Mag. 46 (1923) 671-674.

[45] G.I. Taylor and A.E. Green, Mechanism of the production of small eddies from large ones, Proc. Royal Soc. London Ser. A 158 (1937) 499-521.

[46] M. Visbal and D. Rizzetta, Large-eddy simulation on general geometries using compact differencing and filtering schemes, AIAA Paper (2002) 2002-288.

[47] X. Wang, An efficient second order in time scheme for approximating long time statistical properties of the two dimensional Navier-Stokes equations. Numer. Math. 121 (2012) 753-779.

[48] E. Zeidler, Applied functional analysis, vol. 108 of Appl. Math. Sci. Springer-Verlag, New York (1995). 